\title{
On the Lawrence-Doniach model of superconductivity: magnetic fields parallel to the axes
}

Received March 1, 2010 and in revised form March 10, 2011

\begin{abstract}
We consider periodic minimizers of the Lawrence-Doniach functional, which models highly anisotropic superconductors with layered structure, in the simultaneous limit as the layer thickness tends to zero and the Ginzburg-Landau parameter tends to infinity. In particular, we consider the properties of minimizers when the system is subjected to an external magnetic field applied either tangentially or normally to the superconducting planes. For normally applied fields, our results show that the resulting "pancake" vortices will be vertically aligned. In horizontal fields we show that there are two-parameter regimes in which minimizers exhibit very different characteristics. The low-field regime resembles the Ginzburg-Landau model, while the high-field limit gives a "transparent state" described in the physical literature. To obtain our results we derive sharp matching upper and lower bounds on the global minimizers of the energy.
\end{abstract}

Keywords. Calculus of variations, elliptic equations and systems, superconductivity, vortices

\section{Introduction}

In this paper we study minimizers of the Lawrence-Doniach energy functional in certain asymptotic limits. The Lawrence-Doniach (LD) energy was introduced [LaDo] to model highly anisotropic superconductors having a layered structure. Unlike the GinzburgLandau (GL) model, which represents a superconductor as a continuous three-dimensional solid, in the LD model the superconductor is idealized as a network of equally spaced, parallel superconducting planes. We will consider minimizers of the LD energy under periodic boundary conditions in three dimensions, with applied magnetic fields which are oriented perpendicularly or parallel to these superconducting planes. We study various asymptotic limits, with the distance between the planes and the radius of vortices both tending to zero, for applied magnetic fields which depend on these two parameters. By introducing periodicity we eliminate boundary pinning effects and concentrate on the lower critical field, the value $H_{C 1}$ of the applied field strength at which vortices appear in the superconductor, and how the nature of the vortex lattice is determined by the orientation of the applied field and the relationships between the physical parameters.

S. Alama, L. Bronsard: Department of Mathematics and Statistics, McMaster Univ., Hamilton, Ontario, Canada L8S 4K1; e-mail: alama@mcmaster.ca, bronsard@math.mcmaster.ca

E. Sandier: Département des Mathématiques, Université Paris XII, 64 avenue du Général de Gaulle, 94010 Créteil Cedex, France; e-mail: sandier@u-pec.fr

Mathematics Subject Classification (2010): 35J50, 58J37 
The superconductor occupies an infinite network of parallel planes orthogonal to $\vec{e}_{3}$, with equal spacing $s>0$,

$$
\mathcal{P}=\bigcup_{n \in \mathbb{Z}} \mathcal{P}_{n}, \quad \mathcal{P}_{n}:=\mathbb{R}^{2} \times\{n s\} .
$$

The most general periodic structure on this network fixes three independent vectors, $\vec{v}_{i}$, $i=1,2,3$, for which $\mathcal{P}+k \vec{v}_{i}=\mathcal{P}, i=1,2,3$. However, for our analysis we will assume that one vector lies along the applied field direction. We make this hypothesis for simplicity, but we conjecture that all the results below hold without regard to the geometry of the underlying period domain. Thus, we begin by assuming

$$
\vec{v}_{3}=L_{3} \vec{e}_{3}
$$

and define a fundamental period domain $\Omega \subset \mathbb{R}^{3}$ by fixing a basis $\left\{\vec{v}_{1}, \vec{v}_{2}\right\}$ of vectors in $\mathbb{R}^{2} \subset \mathbb{R}^{3}$,

$$
\Omega:=\left\{t_{1} \vec{v}_{1}+t_{2} \vec{v}_{2}+t_{3} \vec{v}_{3}: t_{j} \in[0,1], j=1,2,3\right\}
$$

When convenient, we will abuse notation and write $\vec{v}_{1}, \vec{v}_{2}$ as elements of either $\mathbb{R}^{2}$ or $\mathbb{R}^{3}$. We denote the restriction of each plane $\mathcal{P}_{n}$ to $\Omega$ by $P_{n}$, that is,

$$
\begin{array}{r}
P_{n}=P+\left(0,0, z_{n}\right), \quad z_{n}:=n s, \quad P:=\left\{t_{1} \vec{v}_{1}+t_{2} \vec{v}_{2}: t_{j} \in[0,1], j=1,2\right\}, \\
n=1, \ldots, N .
\end{array}
$$

On each plane we define a complex-valued order parameter, $u_{n}: \mathcal{P}_{n} \simeq \mathbb{R}^{2} \rightarrow \mathbb{C}$. As in the Ginzburg-Landau model, $\left|u_{n}\right|=1$ represents the purely superconducting state, and $u_{n}=0$ the normal state. The superconducting currents interact with a magnetic field, described by a vector potential $A: \mathbb{R}^{3} \rightarrow \mathbb{R}^{3}$ via $h=\nabla \times A$.

To write the LD energy we introduce some convenient notation. We denote $\nabla^{\prime}=$ $\left(\partial_{x}, \partial_{y}\right)$ and $A^{\prime}=\left(A_{x}(x, y, z), A_{y}(x, y, z)\right), A_{n}^{\prime}=\left(A_{x}\left(x, y, z_{n}\right), A_{y}\left(x, y, z_{n}\right)\right)$. We write $\left(u_{n}, A\right)$ as a shorthand for $\left(\left\{u_{n}\right\}_{n \in \mathbb{Z}}, A\right)$. Then the energy in the period domain $\Omega$ may be written as

$$
\begin{aligned}
\mathcal{L}_{\epsilon, s}^{\lambda}\left(u_{n}, A\right)= & s \sum_{n=1}^{N(s)} \int_{P}\left[\frac{1}{2}\left|\left(\nabla^{\prime}-i A_{n}^{\prime}\right) u_{n}\right|^{2}+\frac{1}{4 \epsilon^{2}}\left(\left|u_{n}\right|^{2}-1\right)^{2}\right] d x d y \\
& +s \sum_{n=1}^{N} \int_{P} \frac{1}{2 \lambda^{2} s^{2}}\left|u_{n}-u_{n-1} e^{i \int_{z_{n-1}}^{z_{n}} A_{z}(x, y, z) d z}\right|^{2} d x d y \\
& +\frac{1}{2} \int_{\Omega}\left|\nabla \times A-\vec{h}_{\mathrm{ex}}\right|^{2} d x d y d z .
\end{aligned}
$$

Here, $\epsilon>0$ represents the reciprocal of the Ginzburg-Landau parameter; we will assume $\epsilon \ll 1$, which is typical for type-II superconducting materials. The constant $\lambda>0$ represents the Josephson penetration depth, and will be assumed to be fixed in this paper. (Results on the large $\lambda$ limit of the LD minimizers may be found in [ABB1, ABB2].) The applied field $\vec{h}_{\mathrm{ex}}$ is a given constant vector, which will in general depend on $s$ or $\epsilon$, and which we will assume to be either parallel to the planes $P_{n}$ or to the $x_{3}$-axis throughout. 
Next we must define a space of functions for $\mathcal{L}_{\epsilon, s}^{\lambda}$. We say $\left(u_{n}, A\right) \in \mathcal{H}$ if $u_{n} \in$ $H_{\mathrm{loc}}^{1}\left(\mathbb{R}^{2} ; \mathbb{C}\right)$ for all $n \in \mathbb{Z}, A \in H_{\mathrm{loc}}^{1}\left(\mathbb{R}^{3} ; \mathbb{R}^{3}\right)$, and there exist functions $\omega_{j} \in H_{\mathrm{loc}}^{2}\left(\mathbb{R}^{3}\right)$, $j=1,2,3$, such that

$$
\left\{\begin{array}{l}
u_{n}\left(x^{\prime}+\vec{v}_{j}\right)=u_{n}\left(x^{\prime}\right) e^{i \omega_{j}\left(x^{\prime}, z_{n}\right)}, \quad j=1,2, n \in \mathbb{Z}, \\
u_{n+N}\left(x^{\prime}\right)=u_{n}\left(x^{\prime}\right) e^{i \omega_{3}\left(x^{\prime}, z_{n}\right)}, \quad n \in \mathbb{Z} \\
A\left(x+v_{j}\right)=A(x)+\nabla \omega_{j}(x), \quad j=1,2,3
\end{array}\right.
$$

for all $x=(x, y, z)=\left(x^{\prime}, z\right) \in \mathbb{R}^{3}$. That is, the configuration $\left(u_{n}, A\right)$ is $\Omega$-periodic up to gauge transformation. In particular, the gauge invariant quantities

$$
\begin{gathered}
j_{n}^{\prime}(x, y)=\operatorname{Im}\left\{\overline{u_{n}}\left(\nabla^{\prime}-i A_{n}^{\prime}\right) u_{n}\right\}, \quad j_{z}(x, y)=-\operatorname{Im}\left\{\overline{u_{n}} u_{n-1} e^{i \int_{z_{n-1}}^{z_{n}} A_{z}(x, y, z) d z}\right\}, \\
\rho_{n}(x, y)=\left|u_{n}\right|, \quad h=\nabla \times A,
\end{gathered}
$$

are all $\Omega$-periodic.

An important consequence of periodic boundary conditions is the exact quantization of the magnetic flux through period planes: see (4.18) in our later discussion of the minimizers in parallel fields.

\section{Perpendicular fields}

We first discuss the case where $\vec{h}_{\mathrm{ex}}$ is perpendicular to the planes $P_{n}$. This is the setting in which the discrete nature of the model is the least apparent. Indeed, in this case we expect the flux lines to be vertically aligned, penetrating each plane in a stack of "pancake" vortices $([\mathrm{Cl}])$ which resemble a two-dimensional GL vortex array in each plane.

First we recall the classical three-dimensional Ginzburg-Landau functional, in our periodic setting. Let $\mathcal{H}_{\mathrm{GL}}$ denote the space of functions $(\psi, A)$ with $\psi \in H_{\mathrm{loc}}^{1}\left(\mathbb{R}^{3}\right), A \in$ $H_{\mathrm{loc}}^{1}\left(\mathbb{R}^{3} ; \mathbb{R}^{3}\right)$ for which there exist $\omega_{j} \in H_{\mathrm{loc}}^{2}\left(\mathbb{R}^{2}\right), j=1,2,3$, with

$$
\psi\left(x+\vec{v}_{j}\right)=\psi(x) e^{i \omega_{j}(x)}, \quad A\left(x+\vec{v}_{j}\right)=A(x)+\nabla \omega_{j}(x), \quad x \in \mathbb{R}^{3}, j=1,2,3 .
$$

Write the Ginzburg-Landau energy as

$$
G_{\epsilon}(\psi, A)=\int_{\Omega}\left[\frac{1}{2}|(\nabla-i A) \psi|^{2}+\frac{1}{4 \epsilon^{2}}\left(|\psi|^{2}-1\right)^{2}+\frac{1}{2}\left|\nabla \times A-h_{\mathrm{ex}}^{\perp} \vec{e}_{3}\right|^{2}\right] .
$$

We prove:

Theorem 1.1. Let $\epsilon>0$ and $\vec{h}_{\mathrm{ex}}=h_{\mathrm{ex}}^{\perp} \vec{e}_{3}$ be given.

1. For any $s=L_{3} / N$, any minimizer of $\mathcal{L}_{\epsilon, s}^{\lambda}$ in $\mathcal{H}$ is gauge equivalent to $\left(u_{n}, A\right)$ with

$$
\left\{\begin{array}{l}
u_{n}(x, y)=u_{n-1}(x, y), \quad A\left(x^{\prime}, z+s\right)=A\left(x^{\prime}, z\right), \\
A_{x}(x, y, s-z)=A_{x}(x, y, z), \quad A_{y}(x, y, s-z)=A_{x}(x, y, z), \quad A_{z}(x, y, z)=0,
\end{array}\right.
$$

for all $(x, y) \in P$ and $n \in \mathbb{Z}$. 
2. If ( $\left.\psi_{\mathrm{GL}}, A_{\mathrm{GL}}\right)$ are minimizers of $G_{\epsilon}$, then

$$
G_{\epsilon}\left(\psi_{\mathrm{GL}}, A_{\mathrm{GL}}\right) \leq G_{\epsilon}\left(u_{n}, A\right) \leq \mathcal{L}_{\epsilon, s}^{\lambda}\left(u_{n}, A\right)(1+2 s) \leq G_{\epsilon}\left(\psi_{\mathrm{GL}}, A_{\mathrm{GL}}\right)(1+2 s) .
$$

3. If in addition we assume the applied field $h_{\mathrm{ex}}^{\perp}$ is $H_{\mathrm{ex}}|\ln \epsilon|$, we have

$$
\lim _{\epsilon, s \rightarrow 0} \frac{\mathcal{L}_{\epsilon, s}^{\lambda}\left(u_{n}, A\right)}{|\Omega||\ln \epsilon|^{2}}=\frac{1}{2} \min _{H \in \mathbb{R}}\left[|H|+\left(H-H_{\mathrm{ex}}\right)^{2}\right] .
$$

Moreover, $h /|\ln \epsilon| \rightarrow H_{*}$ in $L^{2}$, where $H_{*}=\left(H_{\mathrm{ex}}-1 / 2\right)_{+}$is the minimizer of the right-hand side above.

4. If instead $|\ln \epsilon| \ll h_{\mathrm{ex}} \ll \epsilon^{-2}$, then $h / h_{\mathrm{ex}} \rightarrow 1$ in $L^{2}(\Omega)$ and

$$
\lim _{\epsilon, s \rightarrow 0} \frac{\mathcal{L}_{\epsilon, s}^{\lambda}\left(u_{n}, A\right)}{|\Omega| h_{\mathrm{ex}}^{\perp} \ln \frac{1}{\epsilon \sqrt{h_{\mathrm{ex}}^{\perp}}}}=\frac{1}{2} .
$$

Corollary 1.2. We keep the notation $\left(u_{n}, A\right)$ for a minimizer of $\mathcal{L}_{\epsilon, s}^{\lambda}$ and $\left(\Psi_{\mathrm{GL}}, A_{\mathrm{GL}}\right)$ for a minimizer of $G_{\epsilon}$, and we let $h=\nabla \times A$ and $h_{\mathrm{GL}}=\nabla \times A_{\mathrm{GL}}$. Then

1. $h(x, y, z)=\left(-h_{x}(x, y,-z),-h_{y}(x, y,-z), h_{z}(x, y,-z)\right)$ and $h(x, y, z+s)=$ $h(x, y, s)$.

2. If $h_{\mathrm{ex}}^{\perp} \ll \epsilon^{-2}$, then

$$
\lim _{s, \epsilon \rightarrow 0}\left(\frac{h}{h_{\mathrm{ex}}^{\perp}}-\frac{h_{\mathrm{GL}}}{h_{\mathrm{ex}}^{\perp}}\right)=0 \quad \text { in } L^{2}(\Omega) .
$$

Although the vortices will always be vertically aligned, the magnetic field $h=\nabla \times A$ will not be $x_{3}$-invariant. From the Euler-Lagrange equations (see Bauman \& Ko [BaK]), the parallel components $h_{1}, h_{2}$ of the magnetic field are harmonic in the gaps between adjacent superconducting planes, and they satisfy jump conditions at the planes themselves. We expect that the magnetic flux lines will spread between the planes and be pinched together near the pancake vortices in each plane, forming hourglass shapes.

We provide the proofs of these results in Section 3.

\section{Parallel fields}

Next, we discuss the situation when the applied field is parallel to the planes. For this part, we assume $\vec{v}_{j}=L_{j} \vec{e}_{j}, j=1,2,3$, where $\vec{e}_{1}, \vec{e}_{2}, \vec{e}_{3}$ is the standard basis for $\mathbb{R}^{3}$, and $\vec{h}_{\mathrm{ex}}=h_{\mathrm{ex}}^{\|} \vec{e}_{2}$ is directed along the $y$-axis. As opposed to the perpendicular (and obliquely oriented) applied fields, when $\vec{h}_{\text {ex }}$ is parallel to the plane, energy minimizers $\left(u_{n}, A\right)$ will be two-dimensional: $u_{n}=u_{n}(x, z), A=\left(A_{x}(x, z), A_{z}(x, z)\right)$, with $\vec{h}=h(x, z) \vec{e}_{2}$ (this is proven in Lemma 4.1). In Section 4 we define a two-dimensional reduction $\mathcal{H}^{\|}$of the space $\mathcal{H}$ of periodic functions, with fundamental domain $Q=\left[0, L_{1}\right] \times\left[0, L_{3}\right]$, for the functional $\mathcal{L}_{\epsilon, s}^{\lambda}$.

Despite this simplification, the case of parallel fields is the one in which the discreteness of the model is the most evident. The currents which flow in the $x z$-plane orthogonal to the magnetic field are extremely anisotropic: horizontal currents run along the planes (in the $x$-direction) but not in the gaps between the planes, and vertical $z$-direction cur- 
rents (due to Josephson's tunneling effect) are measured in the gaps only, and can jump across the planes, as can the values of the magnetic field (see [ABB1, ABB2, ABS1] for the Euler-Lagrange equations in this case). Since the order parameters $u_{n}$ are not defined between the planes, magnetic flux may penetrate parallel to the planes without loss of superconductivity, and we expect $\left|u_{n}\right| \sim 1$ no matter how large the applied field and the induced currents are. One may think of the vortex cores as being pinned between the planes, where they can carry flux without singularity. As a consequence, the usual Ginzburg-Landau parameter $\epsilon$ plays a minor role in this setting, and the size of the vortices is determined by the interlayer spacing, $s$. We only need the hypothesis that

$$
\epsilon \leq s / \alpha
$$

for some $\alpha>0$.

The behavior of minimizers in the parallel setting is determined by the asymptotic value of the quantity $h_{\mathrm{ex}} s^{2}$. If $h_{\mathrm{ex}} s^{2} \ll 1$, we expect that the mean distance between vortices, which should be on the order of $h_{\mathrm{ex}}^{-1 / 2}$, is much larger than the interplanar spacing $s$. Thus, the discrete nature of the problem can be seen as a small effect and the minimizers will resemble those of the Ginzburg-Landau model, but with s playing the role of $\epsilon$.

Theorem 1.3. Assume (1.2) and that $s^{2} h_{\mathrm{ex}} \ll 1$, and $\left(u_{n}, A\right) \in \mathcal{H}^{\|}$are minimizers of $\mathcal{L}_{\epsilon, s}^{\lambda}$, and $h_{s}=\operatorname{curl} A$.

1. If $h_{\mathrm{ex}} \sim H_{\mathrm{ex}}|\ln s|$ for constant $H_{\mathrm{ex}}>0$, then

$$
\lim _{s \rightarrow 0} \frac{\mathcal{L}_{\epsilon, s}^{\lambda}\left(u_{n}, A\right)}{|Q|(\ln s)^{2}}=\lambda^{-1} \min _{H \in \mathbb{R}} \frac{1}{2}\left[|H|+\left(H-\lambda H_{\mathrm{ex}}\right)^{2}\right] .
$$

Moreover, $h_{s} /|\ln s| \rightarrow H_{*}$ in $L^{2}$, with $H_{*}$ a minimizer of the right-hand side above. In particular, if $H_{\mathrm{ex}} \leq 1 / 2 \lambda$, then $H_{*}=0$.

2. If $|\ln s| \ll h_{\mathrm{ex}} \ll s^{-2}$, we have

$$
\lim _{s \rightarrow 0} \frac{\mathcal{L}_{\epsilon, s}^{\lambda}\left(u_{n}, A\right)}{h_{\mathrm{ex}} \ln \frac{1}{s \sqrt{h_{\mathrm{ex}}}}}=\frac{|Q|}{2 \lambda} \quad \text { and } \quad \frac{h_{s}}{h_{\mathrm{ex}}} \rightarrow 1 \quad \text { in } L^{2} .
$$

In particular, the lower critical field $H_{C 1}^{\|}$equals $\frac{1}{2 \lambda}|\ln s|$ for parallel fields. In fact, when $|\ln s| \ll h_{\mathrm{ex}} \ll s^{-2}$ we show that the energy density of an auxiliary function $\mathcal{M}_{S}(\Psi, A)$ (see $[\mathrm{ABS} 1]$ ) associated to the minimizers converges weakly to a constant multiple of the Lebesgue measure.

The proof of Theorem 1.3 follows the same steps as the corresponding results for the two-dimensional Ginzburg-Landau functional (see [AyS, SS]), by means of an upper bound derived by constructing a configuration with a dense vortex lattice, and the vortex ball construction for the lower bound. The discreteness is eliminated by considering a gauge invariant interpolation of $u_{n}$ to the whole period domain $Q$ and an auxiliary functional introduced in [ABS1].

When the applied field is very large, the minimizers of $\mathcal{L}_{\epsilon, s}^{\lambda}$ are qualitatively different from those of the Ginzburg-Landau model. Assuming $h_{\mathrm{ex}} s^{2} \gg 1$, the distance between vortex cores is too small to ignore discretization, and indeed we expect vortices to lie in 
every gap between the planes. Roughly, minimizers will have magnetic field $h \simeq h_{\mathrm{ex}}^{\|}$everywhere and $\left|u_{n}\right| \simeq 1$ (a "transparent state"; see Bulaevskii \& Clem [BuCm]). Since the planes are effectively one-dimensional, we may then solve for the phases $\phi_{n}, u_{n} \simeq e^{i \phi_{n}(x)}$ exactly from the vector potential $A_{x}\left(x, z_{n}\right)$. As a result, the first and third terms in $\mathcal{L}_{\epsilon, s}^{\lambda}$ roughly vanish, leaving only the Josephson coupling term as the principal contribution to energy, at order $1 / s^{2}$. The only complication with this plan is that the explicit solution to $h=\nabla \times A=h_{\mathrm{ex}}, \phi_{n}^{\prime}=A_{x}\left(x, z_{n}\right)$ may not satisfy the Floquet boundary conditions on $Q$. As was observed in a different limiting regime in [ABB1, ABB2], to obtain the minimum of energy among all periodic configurations, it is necessary to slightly modify either the horizontal period $L_{1}$ or the value of $h_{\mathrm{ex}}^{\|}$in order that the natural period of minimizers be commensurate with the imposed period:

$$
h_{\mathrm{ex}} \in \frac{2 \pi N}{|Q|} \mathbb{Z},
$$

where $N$ is the number of superconducting planes in the period domain $Q$. Since $h_{\mathrm{ex}} \gg$ $s^{-2} \sim N^{2}$, this demands a vanishingly small correction to either $h_{\mathrm{ex}}$ or $L_{1}$.

To state our results we make the following definitions:

$$
\begin{aligned}
\rho(x, z) & =\sum_{n=1}^{N}\left|u_{n}(x)\right| \chi_{\left(z_{n-1}, z_{n}\right]}(z) \\
J_{x}(x, z) & =\sum_{n=1}^{N}\left(i u_{n}(x), u_{n}^{\prime}-i A_{x}\left(x, z_{n}\right)\right) \chi_{\left(z_{n-1}, z_{n}\right]}(z) .
\end{aligned}
$$

In some sense, $\rho$ measures the superconducting density, and $J_{x}$ the horizontal currents, extended to the entire bulk. The interpolating function $\Psi$ used in the previous regime cannot be easily related to the energy density, as the control on the error terms in the finite differences in $x$ is lost in this highly oscillatory limit. We prove:

Theorem 1.4. Let $\left(u_{n}, A\right) \in \mathcal{H}$ be minimizers of $\mathcal{L}_{\epsilon, s}^{\lambda}$ with $s^{2} h_{\mathrm{ex}} \gg 1$ and $\epsilon \ll s$. We have

$$
\frac{|Q|}{s^{2}}+o\left(s^{-2}\right) \leq \mathcal{L}_{\epsilon, s}^{\lambda}\left(u_{n}, A\right) \leq \frac{|Q|}{s^{2}}\left(1+\frac{\pi^{2}}{2 L_{1}^{2}}\right)+o\left(s^{-2}\right) .
$$

Moreover, if in addition we assume that $L_{1}, h_{\mathrm{ex}}$ are chosen such that (1.3) holds, then

$$
\begin{gathered}
\lim _{s \rightarrow 0} s^{2} \mathcal{L}_{\epsilon, s}^{\lambda}\left(u_{n}, A\right)=|Q|, \\
\rho \rightarrow 1, \quad s J_{x} \rightarrow 0 \quad \text { strongly in } L^{2}, \\
s\left\|h_{s}-h_{\mathrm{ex}}\right\|_{L^{2}}=s\left\|\nabla \times A-h_{\mathrm{ex}}\right\|_{L^{2}} \rightarrow 0 .
\end{gathered}
$$

From the above we conclude that, to highest order in $s^{-1}$, the magnetic field associated to minimizers coincides with the applied field, $h_{\mathrm{ex}}$, yet the mean density of superconducting electrons does not diminish with increasing $h_{\mathrm{ex}}$. The leading contribution to the energy comes from the Josephson coupling term in the energy, the energy of horizontal currents 
and of the magnetic field being of lower order in the expansion. As a consequence, the Lawrence-Doniach model has no upper critical field in this asymptotic regime in the parallel setting: $H_{C 2}^{\|}=H_{C 3}^{\|}=\infty$. That is, the minimizers for $h_{\mathrm{ex}} \gg s^{-2}$ are never given by the normal solution, $u_{n}=0$, since $\rho \rightarrow 1$ in $L^{2}$.

The relation (1.3) was also crucial to the results in $[\mathrm{ABB} 1]$. In that paper a different limit is considered, that of the coupling constant $\lambda \rightarrow \infty$ with $\epsilon, s h_{\mathrm{ex}}$ fixed. Nevertheless, (1.3) arises in a similar way, with the applied field and interlayer spacing $s$ selecting a natural frequency of oscillation in $x$. If the artificially imposed period $L_{1}$ is not an exact multiple of the natural frequency $2 \pi N / L_{3} h_{\mathrm{ex}}$ then the vortex lattice structure is frustrated, with a leading order energy cost. Since the periodic problem is intended to simulate the "bulk" properties of a very large material, it seems natural to preserve the condition (1.3) by allowing for a modification in the given period $L_{1}$. Note that for any fixed value $L_{1}$ which we might prescribe as a period, we may choose $\tilde{L}_{1} \in 2 \pi N / L_{3} h_{\mathrm{ex}}$ with $\left|\tilde{L}_{1}-L_{1}\right| \leq 2 \pi N / L_{3} h_{\mathrm{ex}}=2 \pi s /\left(s^{2} h_{\mathrm{ex}}\right)=o(s)$, and thus the change in the period domain is imperceptible even with respect to the vanishingly small distance $s$ between the planes.

We believe that the transparent state described in the second part of Theorem 1.4 should resemble the minimizers in the limit $\lambda \rightarrow \infty$ as studied in [ABB1, ABB2]. To see the more detailed structure of minimizers, a sharper evaluation of the Josephson term is necessary, perhaps after some rescaling in $s$, as a sort of second $\Gamma$-limit of the functional.

Finally, the case where $s^{2} h_{\mathrm{ex}}$ is bounded above and below fits neither of these descriptions. In this case, we would expect that the vortices are separated by a finite number of planes, and so the discretization cannot be ignored, yet the external field should not yet penetrate the gaps completely. Other methods will be required to analyze this regime.

\section{Preliminary results}

In order to assert the existence of energy minimizers we must fix a gauge in which the Lawrence-Doniach energy is coercive. We include some details for completeness, as the three-dimensional periodic problem has not been treated in many papers.

For a constant vector field $\bar{h}=\left(\bar{h}_{x}, \bar{h}_{y}, \bar{h}_{z}\right)$ we choose our representative,

$$
\bar{A}=\frac{1}{2} \bar{h} \times \vec{x}=\frac{1}{2}\left(z \bar{h}_{y}-y \bar{h}_{z}, x \bar{h}_{z}-z \bar{h}_{x}, y \bar{h}_{x}-x \bar{h}_{y}\right) .
$$

We then say $\left(u_{n}, A\right) \in \mathcal{H}_{*}$ if $u_{n} \in H_{\text {loc }}^{1}\left(\mathbb{R}^{2} ; \mathbb{C}\right)$ for all $n \in \mathbb{Z}, A \in H_{\text {loc }}^{1}\left(\mathbb{R}^{3} ; \mathbb{R}^{3}\right)$, and there exists a constant vector $\bar{h} \in \mathbb{R}^{3}$ such that

$$
\left\{\begin{array}{l}
A=\bar{A}+A_{0}, \quad \operatorname{div} A_{0}=0, \quad A_{0}\left(\vec{x}+\vec{v}_{j}\right)=A_{0}(\vec{x}), \quad j=1,2,3, \vec{x} \in \mathbb{R}^{3}, \\
u_{n}\left(\vec{x}^{\prime}+\vec{v}_{j}\right)=u_{n}\left(\vec{x}^{\prime}\right) e^{-i \bar{A}\left(\vec{x}^{\prime}, z_{n}\right) \cdot \vec{v}_{j},} \quad j=1,2, \vec{x}^{\prime} \in \mathbb{R}^{2}, n \in \mathbb{Z}, \\
u_{n+N}\left(\vec{x}^{\prime}\right)=u_{n}\left(\vec{x}^{\prime}\right) e^{-i \bar{A}\left(\vec{x}^{\prime}, z_{n}\right) \cdot \vec{v}_{3}}, \quad \vec{x}^{\prime} \in \mathbb{R}^{2}, n \in \mathbb{Z},
\end{array}\right.
$$

where $\bar{A}$ is associated to $\bar{h}$ as in (2.1). Note that $\left(u_{n}, A\right) \in \mathcal{H}_{*}$ satisfy (1.1) with $\omega_{j}(\vec{x})=$ $-\bar{A}(\vec{x}) \cdot \vec{v}_{j}$ 
Lemma 2.1. (a) For any $\left(u_{n}, A\right) \in \mathcal{H}$ there exists $\gamma \in H_{\text {loc }}^{2}\left(\mathbb{R}^{3}\right)$ such that

$$
\left(u_{n} e^{i \gamma\left(\cdot, z_{n}\right)}, A+\nabla \gamma\right) \in \mathcal{H}_{*} .
$$

Moreover, the constant vector $\bar{h}$ is the average value of $\nabla \times A$ in $\Omega$.

(b) There exists a constant $C_{0}=C_{0}(\Omega)$ such that for any $\left(u_{n}, A\right) \in \mathcal{H}_{*}$,

$$
\|A\|_{H^{1}(\Omega)} \leq C_{0}\|\nabla \times A\|_{L^{2}(\Omega)} .
$$

Proof. First assume $\left(u_{n}, A\right) \in \mathcal{H}$. Let $\bar{h}=|\Omega|^{-1} \int_{\Omega} h$, the (componentwise) average of $h=\nabla \times A$ over the period $\Omega$. Now let $\psi$ be the solution of the periodic problem

$$
\left\{\begin{array}{l}
-\Delta \psi=h-\bar{h} \quad \text { in } \mathbb{R}^{3}, \\
\psi\left(\vec{x}+\vec{v}_{j}\right)=\psi(\vec{x}) \quad \text { in } \mathbb{R}^{3}, j=1,2,3, \\
\int_{\Omega} \psi=0 .
\end{array}\right.
$$

By standard elliptic theory, $\psi$ exists and is unique, $\psi \in H_{\Omega}^{2}$, and there exists a constant $C_{1}=C_{1}(\Omega)$ such that

$$
\|\psi\|_{H^{2}(\Omega)} \leq C_{1}\|h-\bar{h}\|_{L^{2}(\Omega)} \leq C_{1}\|h\|_{L^{2}(\Omega)} .
$$

Since $\bar{A}-A+\nabla \times \psi$ is curl-free, it is the gradient of a function $\gamma$ and thus $\bar{A}+\nabla \times \psi=$ $A+\nabla \gamma$. We let $\tilde{A}=A+\nabla \gamma$ and $\tilde{u}=u \exp (i \gamma)$. Since $\mathcal{H}$ is gauge invariant we have $(\tilde{u}, \tilde{A}) \in \mathcal{H}$, and therefore the existence of functions $\tilde{\omega}_{j}, j=1,2,3$, such that

$$
\left\{\begin{array}{l}
\tilde{u}_{n}\left(x^{\prime}+\vec{v}_{j}\right)=\tilde{u}_{n}\left(x^{\prime}\right) e^{i \tilde{\omega}_{j}\left(x^{\prime}, z_{n}\right)}, \quad j=1,2, n \in \mathbb{Z}, \\
\tilde{u}_{n+N}\left(x^{\prime}\right)=\tilde{u}_{n}\left(x^{\prime}\right) e^{i \tilde{\omega}_{3}\left(x^{\prime}, z_{n}\right)}, \quad n \in \mathbb{Z}, \\
\tilde{A}\left(x+v_{j}\right)=\tilde{A}(x)+\nabla \omega_{j}(x), \quad j=1,2,3 .
\end{array}\right.
$$

From the last identity and the fact that $\bar{A}+\nabla \times \psi$ with $\psi$ periodic we deduce that $\nabla \tilde{\omega}_{j}(x)=\bar{A}\left(x+v_{j}\right)-\bar{A}(x)$ and so $\tilde{\omega}_{j}(x)=\frac{1}{2}\left(\bar{h} \times v_{j}\right) \cdot x+c_{j}$, where $c_{j} \in \mathbb{R}$. We may make $c_{j}=0$ by a further gauge transformation: Let $f(x)$ be the linear function on $\mathbb{R}^{3}$ such that $f\left(v_{j}\right)=-c_{j}, j=1,2,3$, and define $v=\tilde{u} \exp ($ if $), B=\tilde{A}+\nabla f$. Note that $\nabla f$ is a constant vector.

Then $(v, B) \in \mathcal{H}$. Moreover $B$ is of the form $\bar{A}+A_{0}$, where $A_{0}=\nabla \times \psi+\nabla f$ is divergence-free and periodic. Finally

$$
\left\{\begin{array}{l}
v_{n}\left(x^{\prime}+\vec{v}_{j}\right)=v_{n}\left(x^{\prime}\right) e^{i \gamma_{j}\left(x^{\prime}, z_{n}\right)}, \quad j=1,2, n \in \mathbb{Z}, \\
v_{n+N}\left(x^{\prime}\right)=v_{n}\left(x^{\prime}\right) e^{i \gamma_{3}\left(x^{\prime}, z_{n}\right)}, \quad n \in \mathbb{Z}, \\
B\left(x+v_{j}\right)=B(x)+\gamma_{j}(x), \quad j=1,2,3,
\end{array}\right.
$$

where

$$
\gamma_{j}=\tilde{\omega}_{j}-c_{j}=\frac{1}{2}\left(\bar{h} \times v_{j}\right) \cdot x=-\frac{1}{2}(\bar{h} \times x) \cdot v_{j}=-\bar{A}(x) \cdot v_{j}
$$


Therefore $(v, B) \in \mathcal{H}_{*}$ and is gauge equivalent to $(u, A)$. This proves part (a) of the lemma.

Part (b) follows from the decomposition of $A=\bar{A}+A_{0}$ above and the elliptic estimate (2.4).

Given the estimate (2.3) the existence of minimizers for $\mathcal{L}_{\epsilon, s}^{\lambda}$ in $\mathcal{H}_{*}$ follows from the direct method in the calculus of variations.

Proposition 2.2. For any $s, \epsilon>0$ the minimum of $\mathcal{L}_{\epsilon, s}^{\lambda}$ is attained in $\mathcal{H}_{*}$. Moreover, the minimizer satisfies $\left|u_{n}\right| \leq 1$ for all $(x, y) \in \mathbb{R}^{2}$ and $n \in \mathbb{Z}$.

The Euler-Lagrange equations and regularity for solutions is treated in $[\mathrm{BaK}]$, where the Lawrence-Doniach system is studied under natural boundary conditions on a bounded domain $\Omega$. It is straightforward to check that the regularity of solutions stated in Theorem 4.6 there holds in the case of entire solutions as well, i.e. when $\Omega=\mathbb{R}^{3}$, and thus in our setting.

Remark 2.3. The estimate $\left|u_{n}\right| \leq 1$ clearly holds for minimizers of the LawrenceDoniach energy in $\mathcal{H}$, since it is easy to check that replacing $u_{n}$ by $u_{n} /\left|u_{n}\right|$ whenever $\left|u_{n}\right|>1$ reduces the energy. It is also true for solutions, following the proof of [BaK] which carries over to the case of periodic boundary conditions. This property is mentioned in [DGP] for the periodic Ginzburg-Landau model as a straightforward extension of the result for the Ginzburg-Landau model with natural boundary conditions.

Finally, we note that analogous spaces for the Ginzburg-Landau functional in the three-dimensional periodic setting may be defined as in (1.1), (2.2). For instance, the Floquet boundary conditions may be stated as

$$
\left\{\begin{array}{l}
u\left(\vec{x}+\vec{v}_{j}\right)=u(\vec{x}) e^{i \omega_{j}(\vec{x})}, \quad j=1,2,3 \\
A\left(\vec{x}+\vec{v}_{j}\right)=A(\vec{x})+\nabla \omega_{j}(\vec{x}), \quad j=1,2,3
\end{array}\right.
$$

for $\omega_{j} \in H_{\mathrm{loc}}^{2}\left(\mathbb{R}^{3} ; \mathbb{R}\right), j=1,2,3$.

\section{Applied field orthogonal to the planes}

We assume now that

$$
\vec{h}_{\mathrm{ex}}=h_{\mathrm{ex}}^{\perp} \vec{e}_{3} .
$$

Our goal is to show that energy minimization chooses an $s$-periodic configuration in $z$, that is, the vortices are vertically aligned and have identical profiles in each plane and gap.

To prove Theorem 1.1 we introduce a functional defined on only one superconducting planar domain $P$ and in half of a gap, $T^{+}=P \times[0, s / 2]$. We consider configurations which are periodic with respect to the plane $P$, and free in $z$. Later on, the minimizers in the entire gap will be obtained by reflection. We denote by $H_{\text {loc+ }}^{1}$ the space of functions 
which are in $H^{1}\left([-R, R]^{2} \times[0, s / 2]\right)$ for every $R \geq 1$. Then we define the space $\mathcal{H}^{+}$to consist of those pairs $(v, A)$ with $v \in H_{\mathrm{loc}}^{1}\left(\mathbb{R}^{2} ; \mathbb{C}\right)$ and $A \in H_{\mathrm{loc}+}^{1}$ such that there exist $\omega_{1}, \omega_{2} \in H_{\mathrm{loc}+}^{2}$ with

$$
\begin{cases}v\left(x^{\prime}+\vec{v}_{1}^{\prime}\right)=v\left(x^{\prime}\right) e^{i \omega_{1}}, & A\left(x+\vec{v}_{1}\right)=A(x)+\nabla \omega_{1}(x), \\ v\left(x^{\prime}+\vec{v}_{2}^{\prime}\right)=v\left(x^{\prime}\right) e^{i \omega_{2}}, & A\left(x+\vec{v}_{2}\right)=A(x)+\nabla \omega_{2}(x),\end{cases}
$$

for all $x^{\prime} \in \mathbb{R}^{2}, x \in \mathbb{R}^{2} \times[0, s / 2]$. We define a functional $F^{+}$for $(v, A) \in \mathcal{H}^{+}$,

$$
\begin{aligned}
F^{+}(v, A)= & \frac{s}{2} \int_{P}\left[\frac{1}{2}\left|\left(\nabla^{\prime}-i A^{\prime}(x, y, 0)\right) v\right|^{2}+\frac{1}{4 \epsilon^{2}}\left(|v|^{2}-1\right)^{2}\right] d x d y \\
& +\frac{1}{2} \int_{T^{+}}\left|\nabla \times A-\vec{h}_{\mathrm{ex}}\right|^{2} d x d y d z .
\end{aligned}
$$

As we did for the fully periodic problem we define a subspace $\mathcal{H}_{*}^{+}$which fixes a Coulomb-type gauge in $\mathcal{H}^{+}$. Given a constant $\bar{h}_{z} \in \mathbb{R}$, let

$$
\bar{A}=\frac{\bar{h}_{z}}{2}(-y, x, 0) \text {. }
$$

We say $(v, A) \in \mathcal{H}_{*}^{+}$if $v \in H_{\mathrm{loc}}^{1}\left(\mathbb{R}^{2} ; \mathbb{C}\right)$ and $A \in H_{\text {loc }+}^{1}$ are such that there exists a constant $\bar{h}_{z} \in \mathbb{R}$ so that (recall $\vec{v}_{3}=L_{3} \vec{e}_{3}$ )

$$
\left\{\begin{array}{l}
A(\vec{x})=\bar{A}+A_{0}, \quad \operatorname{div} A_{0}=0, \quad A_{0}\left(\vec{x}+\vec{v}_{j}\right)=A_{0}(\vec{x}) \quad \text { in } T^{+}, j=1,2, \\
A \cdot \vec{e}_{3}=0 \quad \text { on }\{z=0\} \cup\{z=s / 2\}, \\
v\left(x^{\prime}+\vec{v}_{j}^{\prime}\right)=v\left(x^{\prime}\right) e^{-i \bar{A} \cdot \vec{v}_{j}^{\prime}}, \quad j=1,2, x^{\prime} \in \mathbb{R}^{2} .
\end{array}\right.
$$

Lemma 3.1. (a) For any $(v, A) \in \mathcal{H}^{+}$there exists $\gamma \in H_{\mathrm{loc}}^{2}\left(T^{+}\right)$such that

$$
\left(v e^{i \gamma\left(\cdot, z_{n}\right)}, A+\nabla \gamma\right) \in \mathcal{H}_{*}^{+} .
$$

(b) There exists a constant $C_{0}$ such that for any $\left(u_{n}, A\right) \in \mathcal{H}_{*}^{+}$,

$$
\|A\|_{H^{1}\left(T^{+}\right)} \leq C_{0}\|\nabla \times A\|_{L^{2}\left(T^{+}\right)} .
$$

Proof. The proof follows the same lines as that of Lemma 2.1, except that $\psi$ should solve

$$
\left\{\begin{array}{l}
-\Delta \psi=h-\bar{h}_{3} \vec{e}_{3} \quad \text { in } \mathbb{R}^{2} \times[0, s / 2], \\
\psi\left(\vec{x}+\vec{v}_{j}\right)=\psi(\vec{x}) \quad \text { in } \mathbb{R}^{3}, j=1,2, \\
\psi_{1}=\psi_{2}=0 \quad \text { if } x_{3}=0 \text { or } x_{3}=s / 2, \\
\frac{\partial \psi_{3}}{\partial x_{3}}=0 \quad \text { if } x_{3}=0 \text { or } x_{3}=s / 2, \\
\int_{P \times[0, S / 2]} \psi_{3}=0 .
\end{array}\right.
$$


This is essentially a mixed periodic and Dirichlet problem for $\psi_{1}, \psi_{2}$, and a mixed periodic and Neumann problem for $\psi_{3}$; see the appendix of [BBO] for a general theory of existence and uniqueness for such problems. In this case the geometry of the problem is simple, and existence is a straightforward application of the Riesz representation theorem in the space of $H^{1}$ vector fields with $\psi_{1}=\psi_{2}=0$ on the lateral boundaries of the strip and periodic in $x, y$. As before, we set $A_{0}=\nabla \times \psi$ and note that $A_{0} \cdot v= \pm \nabla \times \psi \cdot \vec{e}_{3}=0$ on the lateral boundaries by the Dirichlet condition. The rest of the proof is exactly as in Lemma 2.1 and is omitted.

The space $\mathcal{H}_{*}^{+}$fixes a Coulomb gauge for $A$, and hence the existence of minimizers $\left(v^{+}, A^{+}\right) \in \mathcal{H}^{+}$of $F^{+}$follows from the direct method by gauge transforming a minimizing sequence so that it lies in $\mathcal{H}_{*}^{+}$, and yields a minimizer which is in $\mathcal{H}_{*}^{+}$. By the regularity theory proved by Bauman \& Ko for the Lawrence-Doniach system we have $v^{+}$smooth on $P, A^{+} \in C^{1, \alpha}\left(T^{+}\right)$, and in fact (note that $\Delta h^{+}=0$ in the interior of $T^{+}$), $A^{+}$is smooth on $P \times(0, s / 2]$.

We now show that the minimizers of $F^{+}$on $\mathcal{H}^{+}$may be reflected to obtain (symmetric) energy minimizers in each (full) gap. To see this, we define a space $\mathcal{H}^{-}$of configurations in the lower half-gap, $T^{-}=P \times[-s / 2,0]$. The analogous functional $F^{-}(v, A)$ is defined as in (3.1), with the integral now over $T^{-}$. Again, the minimizer of $F^{-}$over $\mathcal{H}^{-}$ is attained. In fact, for any $(v, A) \in \mathcal{H}^{+}$, define $(\tilde{v}, \tilde{A})$ in $T^{-}$by $\tilde{v}=v$ and

$$
\tilde{A}(x, y, z)=\left(A_{x}(x, y,-z), A_{y}(x, y,-z),-A_{z}(x, y,-z)\right), \quad z \in[-s / 2,0] .
$$

It is easy to see that $(\tilde{v}, \tilde{A}) \in \mathcal{H}^{-}$. Moreover, we have

$$
\begin{array}{r}
\tilde{h}(x, y, z)=\nabla \times \tilde{A}(x, y, z)=\left(-h_{x}(x, y,-z),-h_{y}(x, y,-z), h_{z}(x, y,-z)\right), \\
z \in[-s / 2,0],
\end{array}
$$

and hence

$$
\begin{aligned}
\left|\nabla \times \tilde{A}(x, y, z)-\vec{h}_{\mathrm{ex}}\right|^{2} & =\left|\nabla \times \tilde{A}-h_{\mathrm{ex}}^{\perp} \vec{k}\right|^{2}=\left|h_{x}\right|^{2}+\left|h_{y}\right|^{2}+\left|h_{z}-h_{\mathrm{ex}}^{\perp}\right|^{2} \\
& =\left|\nabla \times A(x, y,-z)-\vec{h}_{\mathrm{ex}}\right|^{2} .
\end{aligned}
$$

In particular, $F^{-}(\tilde{v}, \tilde{A})=F^{+}(v, A)$, and therefore

$$
I:=\min _{(v, A) \in \mathcal{H}^{+}} F^{+}(v, A)=\min _{(v, A) \in \mathcal{H}^{-}} F^{-}(v, A) .
$$

Next, we use the minimizers of $F^{ \pm}$as building blocks for a configuration $\left(u_{n}, A\right)$. Let $\left(v^{+}, A^{+}\right) \in \mathcal{H}_{*}^{+}$be a minimizer of $F^{+}$. By the above remarks, $\left(v^{+}, \tilde{A}^{+}\right) \in \mathcal{H}_{*}^{-}\left(\right.$with $\tilde{A}^{+}$ constructed as in (3.3)) are minimizers of $F^{-}$. Moreover, by odd/even symmetry and the boundary condition of (3.2), we note that the extension $\hat{A}$ defined by

$$
\hat{A}(x, y, z)= \begin{cases}A^{+}(x, y, z) & \text { for }(x, y, z) \in T^{+}, \\ \tilde{A}^{+}(x, y, z) & \text { for }(x, y, z) \in T^{-}\end{cases}
$$


is continuous across the plane $P$, and therefore $\hat{A} \in H_{\text {per }}^{1}\left(P \times[-s / 2, s / 2] ; \mathbb{R}^{2}\right)$. We remark that $\hat{A}_{z}$ is odd in $z$, and hence

$$
\int_{-s / 2}^{s / 2} \hat{A}_{z} d z=0
$$

Continuing, the symmetries of $\hat{A}$ permit us to extend $\hat{A}$ to all of $\mathbb{R}^{3}$ as an $s$-periodic $H^{1}$ (in fact, continuous) function $A^{*}$ in $z$,

$$
A^{*}(x, y, z+n s)=\hat{A}(x, y, z), \quad(x, y) \in P, z \in[-s / 2, s / 2], n \in \mathbb{Z} .
$$

From our observation (3.4) we conclude that

$$
\int_{z_{n-1}}^{z_{n}} A_{z}^{*} d z=0, \quad \forall n
$$

We also define $u_{n}^{*}=v^{+}$for all planes $n=0, \ldots, N(s)-1$. It is easy to see that $\left(u_{n}^{*}, A^{*}\right) \in \mathcal{H}$.

The configuration $\left(u_{n}^{*}, A^{*}\right) \in \mathcal{H}$ gives us an upper bound on the Lawrence-Doniach energy. Indeed, notice that since $u_{n}^{*}=u_{m}^{*}$ for all $n, m$ and by (3.5) the Josephson interaction term is identically zero,

$$
u_{n}^{*}(x, y)-u_{n-1}^{*}(x, y) e^{i \int_{z_{n-1}}^{z n} A_{z}^{*}(x, y, z) d z} \equiv 0, \quad \forall(x, y) \in P, n=1, \ldots, N(s) .
$$

Therefore, the energy of this configuration exactly decouples into $N(s)$ copies of $F^{+}$ and $F^{-}$, and this gives the upper bound

$$
\begin{aligned}
\min _{\mathcal{H}_{*}} \mathcal{L}_{\epsilon, s}^{\lambda} \leq \mathcal{L}_{\epsilon, s}^{\lambda}\left(u_{n}^{*}, A^{*}\right) & =\sum_{n=1}^{N(s)}\left(F^{+}\left(u_{n}^{*}, A^{*}(\cdot, \cdot, z-n s)\right)+F^{-}\left(u_{n}^{*}, A^{*}(\cdot, \cdot, z-n s)\right)\right) \\
& =2 N(s) I .
\end{aligned}
$$

We now show that the Lawrence-Doniach energy is also bounded below by this same quantity. Indeed, for any configuration $\left(u_{n}, A\right) \in \mathcal{H}$, the restriction to each half-gap $T^{ \pm}$ gives an element of $\mathcal{H}^{ \pm}$. We then observe that

$$
\mathcal{L}_{\epsilon, s}^{\lambda}\left(u_{n}, A\right) \geq \sum_{n=1}^{N(s)}\left(F^{+}\left(u_{n}, A(\cdot, \cdot, z-n s)\right)+F^{-}\left(u_{n}, A(\cdot, \cdot, z-n s)\right)\right) \geq 2 N(s) I .
$$

Therefore, $\min _{\mathcal{H}} \mathcal{L}_{\epsilon, s}^{\lambda}=2 N(s) I=\mathcal{L}_{\epsilon, s}^{\lambda}\left(u_{n}^{*}, A^{*}\right)$. In particular, the configuration $\left(u_{n}^{*}, A^{*}\right)$ with vertically aligned vortices is a global minimizer of $\mathcal{L}_{\epsilon, s}^{\lambda}$ for all values of the parameters.

Now we claim that any minimizer $\left(u_{n}, A\right)$ of $\mathcal{L}_{\epsilon, S}^{\lambda}$ is gauge equivalent to one of the above form, with vertically aligned vortices and with the desired symmetries. First, after a gauge transformation, we may assume $\left(u_{n}, A\right) \in \mathcal{H}_{*}$, thus in particular from the regularity result in $\left[\mathrm{BaK}\right.$, Theorem 4.6], we have $A_{z} \in H_{\mathrm{loc}}^{2}\left(\mathbb{R}^{3}\right)$. Then, the tightness of 
the upper and lower bounds on the energy imposes two conclusions. First, the Josephson coupling term must vanish, and hence (3.6) holds for any minimizer $\left(u_{n}, A\right)$. By making the gauge transformation with $\varphi(x, y, z)=-\int_{0}^{z} A_{z}\left(x, y, z^{\prime}\right) d z^{\prime}$ - which is in $H_{\mathrm{loc}}^{2}$ as noted above—we may assume our minimizer satisfies

$$
u_{n}=u_{n-1}=: u \quad \text { for all } n \in \mathbb{Z}, \quad A_{z}(x, y, z)=0 .
$$

In particular, the vortices are vertically aligned, and the currents are identical in each superconducting plane. To arrive at a second conclusion from the energy identity, define

$$
A_{n}^{ \pm}(x, y, z):=\left.A(x, y, z+n s)\right|_{T^{ \pm}}, \quad n \in \mathbb{Z},
$$

the restriction of the minimizing potential $A$ to a half-gap around $P_{n}$. Then $\left(u, A_{n}^{ \pm}\right) \in \mathcal{H}_{*}^{ \pm}$, and minimizes $F^{ \pm}$for each $n \in \mathbb{Z}$. Thus, we observe that $A_{n}^{ \pm}$also minimizes the functional $f^{ \pm}(B)=F^{ \pm}(u, B)$ among $B$ obeying the conditions (3.2), with given $u=u_{n}$. Since $f^{ \pm}(B)$ is quadratic and coercive on such $B$, the minimizer is unique, and thus $A_{n}^{ \pm}$ is the same for each $n \in \mathbb{Z}$, that is, $A(x, y, z+s)=A(x, y, z)$ in this gauge. To recover the other symmetries of the minimizer, we again define the reflection $\tilde{A}$ as in (3.3). As above, we see that $f^{-}(\tilde{A})=f^{+}(A)$ uniquely attains the minimum value among vector potentials obeying (3.2). Thus, $\tilde{A}=A$ in the strip $z \in[-s / 2,0]$. In particular, the magnetic field satisfies

$$
h(x, y, z)=\left(-h_{x}(x, y,-z),-h_{y}(x, y,-z), h_{z}(x, y,-z)\right), \quad z \in[-s / 2,0] .
$$

This completes the proof of part 1 of Theorem 1.1 .

Next we compare the energy of minimizers of $\mathcal{L}_{\epsilon, s}^{\lambda}$ with those of the Ginzburg-Landau model. Assume $\left(\psi_{\mathrm{GL}}, A_{\mathrm{GL}}\right)$ is a $\Omega$-periodic minimizer of the Ginzburg-Landau functional with vertical applied field,

$$
G_{\epsilon}(\psi, A)=\int_{\Omega}\left[\frac{1}{2}|(\nabla-i A) \psi|^{2}+\frac{1}{4 \epsilon^{2}}\left(|\psi|^{2}-1\right)^{2}+\frac{1}{2}\left|h-h_{\mathrm{ex}}^{\perp} \vec{e}_{3}\right|^{2}\right],
$$

on the space $\mathcal{H}_{\mathrm{GL}}$ incorporating the Floquet boundary conditions (see (2.5)). The minimizers in this geometry are two-dimensional, that is, they are gauge equivalent to $\psi_{\mathrm{GL}}=$ $\psi_{\mathrm{GL}}(x, y), A_{\mathrm{GL}}=A_{\mathrm{GL}}^{\prime}(x, y)$. Choosing this special gauge, we define a test configuration for Lawrence-Doniach, $\hat{u}_{n}=\psi_{\mathrm{GL}}(x, y), n=1, \ldots, N$, and $\hat{A}=A_{\mathrm{GL}}$. We then clearly have

$$
\min \mathcal{L}_{\epsilon, s}^{\lambda} \leq \mathcal{L}_{\epsilon, s}^{\lambda}\left(\hat{u}_{n}, \hat{A}\right)=G_{\epsilon}\left(\psi_{\mathrm{GL}}, A_{\mathrm{GL}}\right)
$$

valid for all $s, \epsilon>0$.

To produce a matching lower bound, let $\left(u_{n}, A\right) \in \mathcal{H}_{*}$ be minimizers of $\mathcal{L}_{\epsilon, s}^{\lambda}$. By the preceding analysis and after a gauge transformation, $u_{n}=u_{n-1}=u$ for each $n$. In each gap we define

$$
\Psi(x, y, z)=u(x, y) e^{-i \int_{z}^{z_{n}} A_{z}\left(x, y, z^{\prime}\right) d z^{\prime}}, \quad z_{n-1} \leq z<z_{n} .
$$


By (3.5), the exponent vanishes at each plane $z=z_{n}$, and so $\Psi$ is continuous across the planes. Furthermore, the configuration $(\Psi, A) \in \mathcal{H}_{\mathrm{GL}}$ satisfies Floquet boundary conditions on the solid domain $\Omega$ (with the same $\omega_{j}$ as $u_{n}$ ). Clearly we have

$$
\left|\left(\partial_{z}-i A_{z}(x, y, z)\right) \Psi\right|^{2}=0 .
$$

For the other derivatives we calculate

$$
\begin{aligned}
\int_{P} \int_{z_{n-1}}^{z_{n}} & \left|\left(\partial_{x}-i A_{x}(x, y, z)\right) \Psi\right|^{2}=\int_{P} \int_{z_{n-1}}^{z_{n}}\left|\left(\partial_{x}-i A_{x}(x, y, z)\right) u-i u \int_{z}^{z_{n}} \partial_{x} A_{z} d z^{\prime}\right|^{2} \\
& =\int_{P} \int_{z_{n-1}}^{z_{n}}\left|\left(\partial_{x}-i A_{x}(x, y, z)\right) u-i u \int_{z}^{z_{n}}\left[\partial_{z} A_{x}-h_{y}\right] d z^{\prime}\right|^{2} \\
& =\int_{P} \int_{z_{n-1}}^{z_{n}}\left|\left(\partial_{x}-i A_{x}\left(x, y, z_{n}\right)\right) u+i u \int_{z}^{z_{n}} h_{y} d z^{\prime}\right|^{2} \\
& \leq(1+s) \int_{P} \int_{z_{n-1}}^{z_{n}}\left|\left(\partial_{x}-i A_{x}\left(x, y, z_{n}\right)\right) u\right|^{2}+\left(1+s^{-1}\right) s \int_{P}^{z_{z_{n-1}}}\left[\int_{z_{n-1}}^{z_{n}} h_{y}^{2} d z^{\prime}\right] \\
& =(1+s) \int_{P} \int_{z_{n-1}}^{z_{n}}\left|\left(\partial_{x}-i A_{x}\left(x, y, z_{n}\right)\right) u\right|^{2}+2 s \int_{P}^{z_{n}} h_{z_{n-1}}^{2} .
\end{aligned}
$$

An analogous estimate holds for $\left(\partial_{y}-i A_{y}\right) \Psi$, in terms of the $L^{2}$ norm of $h_{x}$ in the gap. Summing over the gaps we have

$$
\int_{\Omega}\left|\left(\nabla^{\prime}-i A^{\prime}(x, y, z)\right) \Psi\right|^{2} \leq(1+s) s \sum_{n=1}^{N} \int_{P_{n}}\left|\left(\nabla^{\prime}-i A^{\prime}\right) u_{n}\right|^{2}+2 s \int_{\Omega}\left(h_{x}^{2}+h_{y}^{2}\right) .
$$

As each of these terms on the right-hand side appear in the Lawrence-Doniach energy, we arrive at

$$
G_{\epsilon}\left(\psi_{\mathrm{GL}}, A_{\mathrm{GL}}\right) \leq G_{\epsilon}(\Psi, A) \leq(1+2 s) \mathcal{L}_{\epsilon, s}^{\lambda}\left(u_{n}, A\right) \leq(1+2 s) G_{\epsilon}\left(\psi_{\mathrm{GL}}, A_{\mathrm{GL}}\right)
$$

via (3.7). This proves part 2 of Theorem 1.1.

To verify part 3, we assume $h_{\mathrm{ex}}^{\perp}=H_{\mathrm{ex}}^{\perp}|\ln \epsilon|$. From Aydi [AyS] (see also [ABS2] for the three-dimensional setting), in this regime the energy of periodic GL minimizers satisfies

$$
\lim _{\epsilon \rightarrow 0} \frac{G_{\epsilon}\left(\psi_{\mathrm{GL}}, A_{\mathrm{GL}}\right)}{|\Omega||\ln \epsilon|^{2}}=\min _{H \in \mathbb{R}} \frac{1}{2}\left[|H|+\left(H-H_{\mathrm{ex}}^{\perp}\right)^{2}\right] .
$$

Since (3.8) holds uniformly in $\epsilon$, we conclude that

$$
\lim _{\epsilon, s \rightarrow 0} \frac{\mathcal{L}_{\epsilon, s}^{\lambda}\left(u_{n}, A\right)}{|\Omega||\ln \epsilon|^{2}}=\min _{H \in \mathbb{R}} \frac{1}{2}\left[|H|+\left(H-H_{\mathrm{ex}}^{\perp}\right)^{2}\right] .
$$

Moreover, still from [AyS] or [ABS2], there is convergence of curl $A /|\ln \epsilon|$ to the minimizer $H_{*}$ of the right-hand side. We conclude that, in the simultaneous limit $\epsilon, s \rightarrow 0$, 
the two models give rise to the same lower critical field $H_{C 1}=H_{C 1}^{\perp}=\frac{1}{2}|\ln \epsilon|$, meaning more precisely that if $H_{\mathrm{ex}}^{\perp}<1 / 2$ then $H_{*}=0$ while if $H_{\mathrm{ex}}^{\perp}>1 / 2$ then $H_{*} \neq 0$.

Next consider $h_{\mathrm{ex}}^{\perp}$ with $|\ln \epsilon| \ll h_{\mathrm{ex}}^{\perp} \ll \epsilon^{-2}$. Since the minimizers of $G_{\epsilon}$ are twodimensional, we may apply Theorem 8.1 of [SS] which gives the asymptotic limit of minimizers of the Ginzburg-Landau functional in this regime. Again using the estimate (3.8) we obtain part 4 of Theorem 1.1.

This concludes the proof of Theorem 1.1. The statements in Corollary 1.2 follow immediately.

\section{Applied field parallel to the planes}

We recall that in this part, we assume $\vec{v}_{j}=L_{j} \vec{e}_{j}, j=1,2,3$, where $\vec{e}_{1}, \vec{e}_{2}, \vec{e}_{3}$ is the standard basis for $\mathbb{R}^{3}$, and we consider applied fields parallel to the planes,

$$
\vec{h}_{\mathrm{ex}}=h_{\mathrm{ex}} \vec{e}_{2} \text {. }
$$

We first observe that for parallel fields the problem of minimizing $\mathcal{L}_{\epsilon, s}^{\lambda}$ reduces to a twodimensional one. Indeed, define the two-dimensional LD energy of $v_{n}=v_{n}(x), B=$ $\left(B_{x}(x, z), B_{z}(x, z)\right)$ as

$$
\begin{aligned}
\mathcal{L}_{\epsilon, S}^{\lambda, 2 D}\left(v_{n}, B\right)= & s \sum_{n=1}^{N} \int_{0}^{L_{1}}\left[\frac{1}{2}\left|\left(\partial_{x}-i B_{x}\left(x, z_{n}\right)\right) v_{n}\right|^{2}+\frac{1}{4 \epsilon^{2}}\left(\left|v_{n}\right|^{2}-1\right)^{2}\right] d x \\
& +s \sum_{n=1}^{N} \int_{0}^{L_{1}} \frac{1}{2 \lambda^{2} s^{2}}\left|v_{n}-v_{n-1} e^{i \int_{z_{n-1}}^{z_{n}} B_{z}(x, z) d z}\right|^{2} d x \\
& +\frac{1}{2} \int_{0}^{L_{3}} \int_{0}^{L_{1}}\left|\partial_{z} B_{x}-\partial_{x} B_{z}-h_{\mathrm{ex}}\right|^{2} d x d z .
\end{aligned}
$$

Lemma 4.1. Assume $\epsilon, s>0$ are fixed, and $\vec{h}_{\mathrm{ex}}=h_{\mathrm{ex}} \vec{e}_{2}$ is any fixed parallel external field. If $\left(u_{n}, A\right) \in \mathcal{H}$ is a minimizer of $\mathcal{L}_{\epsilon, s}^{\lambda}$ then it is gauge equivalent to $\left(v_{n}, B\right) \in \mathcal{H}$ with $v_{n}=v_{n}(x), B=\left(B_{x}, 0, B_{z}\right)(x, z)$ a minimizer of $\mathcal{L}_{\epsilon, s}^{\lambda, 2 D}$.

Proof. Let $\left(u_{n}, A\right)$ be minimizers of $\mathcal{L}_{\epsilon, s}^{\lambda}$ (for $s, \epsilon$ fixed and $\vec{h}_{\text {ex }}$ satisfying (4.1) also fixed). If $h_{x}, h_{z} \equiv 0$ and $\left(\partial_{y}-i A_{y}\right) u_{n} \equiv 0$ then $\left(u_{n}, A\right)$ is gauge equivalent to some $\left(v_{n}, B\right)$ as above. Indeed, we may always choose a gauge in which $A_{y}=0$.

Assume for contradiction that this is not the case. Given $y \in \mathbb{R}$, we write $u_{n}^{y}=$ $u_{n}(\cdot, y), A^{y}=\left(A_{x}\left(\cdot, y_{0}, \cdot\right), A_{z}(\cdot, y, \cdot)\right)$. Then, noting that

$$
\mathcal{L}_{\epsilon, s}^{\lambda}\left(u_{n}, A\right)=\int_{0}^{L_{2}} \mathcal{L}_{\epsilon, s}^{\lambda, 2 \mathrm{D}}\left(u_{n}^{y}, A^{y}\right) d y+\frac{s}{2} \sum_{n} \int_{P}\left|\left(\partial_{y}-i A_{y}\right) u_{n}\right|^{2}+\frac{1}{2} \int_{\Omega}\left(h_{x}^{2}+h_{z}^{2}\right),
$$

we deduce that

$$
\int_{0}^{L_{2}} \mathcal{L}_{\epsilon, s}^{\lambda, 2 \mathrm{D}}\left(u_{n}^{y}, A^{y}\right) d y<\mathcal{L}_{\epsilon, s}^{\lambda}\left(u_{n}, A\right)
$$


with strict inequality. Thus there exists $y_{0}$ such that

$$
L_{2} \mathcal{L}_{\epsilon, s}^{\lambda, 2 \mathrm{D}}\left(u_{n}^{y_{0}}, A^{y_{0}}\right)<\mathcal{L}_{\epsilon, s}^{\lambda}\left(u_{n}, A\right) .
$$

We have, writing $v_{n}=u_{n}^{y_{0}}, B=A^{y_{0}}$,

$$
L_{2} \mathcal{L}_{\epsilon, s}^{\lambda, 2 \mathrm{D}}\left(v_{n}, B\right)<\mathcal{L}_{\epsilon, s}^{\lambda}\left(u_{n}, A\right)=\min \mathcal{L}_{\epsilon, s}^{\lambda}
$$

On the other hand we may let $\tilde{v}_{n}(x, y)=v_{n}(x)$ and $\tilde{B}(x, y, z)=\left(B_{x}(x, z), 0, B_{z}(x, z)\right)$. Then

$$
\min \mathcal{L}_{\epsilon, s}^{\lambda} \leq \mathcal{L}_{\epsilon, s}^{\lambda}\left(\tilde{v}_{n}, \tilde{B}\right)=L_{2} \mathcal{L}_{\epsilon, s}^{\lambda, 2 \mathrm{D}}\left(v_{n}, B\right)<\min \mathcal{L}_{\epsilon, s}^{\lambda}
$$

hence a contradiction.

In the remainder of the paper we denote $\mathcal{L}_{\epsilon, s}^{\lambda, 2 \mathrm{D}}=\mathcal{L}_{\epsilon, s}^{\lambda}$ for simplicity.

As was noted in [ABS1], in a purely two-dimensional setting the anisotropy $\lambda$ may be removed by an appropriate change of variables. This is not the case in the fully threedimensional setting, where anisotropy plays an essential role in the geometry of minimizers: see [ABS2]. Here we make the simple rescaling,

$$
\tilde{x}=x / \lambda, \quad \tilde{z}=z, \quad \tilde{A}_{x}=\lambda A_{x}, \quad \tilde{A}_{z}=A_{z} .
$$

Then, for any configuration $\left(u_{n}, A\right) \in \mathcal{H}(Q)$, we have $\left(\tilde{u}_{n}, \tilde{A}\right) \in \mathcal{H}\left(\left[0, L_{1} / \lambda\right] \times\left[0, L_{3}\right]\right)$ with

$$
\mathcal{L D}_{\epsilon / \lambda, s}^{1}\left(\tilde{u}_{n}, \tilde{A} ;\left[0, L_{1} / \lambda\right] \times\left[0, L_{3}\right] ; \lambda h_{\mathrm{ex}}\right)=\lambda \mathcal{L} \mathcal{D}_{\epsilon, s}^{\lambda}\left(u_{n}, A ; Q ; h_{\mathrm{ex}}\right) .
$$

In the following we may then suppose $\lambda=1$, and adjust our results for the general case accordingly.

We now distinguish two different parameter regimes, corresponding to two very different behaviors for minimizers.

Case $s^{2} h_{\mathrm{ex}} \ll 1$

In this case, the distance between vortices is $h_{\mathrm{ex}}^{-1 / 2} \gg s$, the interlayer spacing, and we will see that the vortices resemble Ginzburg-Landau vortices to leading order but with the rôle of $\epsilon$ replaced by $s$. In this regime we will prove Theorem 1.3 from the introduction.

We begin by deriving an upper bound for the energy of minimizers. This upper bound is a modification of the more standard upper bound for periodic two-dimensional configuration for the 2D Ginzburg-Landau energy. We provide some details for the standard case since we will need to modify slightly the construction and also obtain more precise estimates. Similar constructions can be found in earlier papers (see for example Section 3 in [AyS] or Proposition 8.1 of [SS] for item 2 of Proposition 4.2 below). Here we present a proof that includes both the cases where $h_{\mathrm{ex}}=H_{\mathrm{ex}}|\ln \epsilon|$ and where $|\ln \epsilon| \ll h_{\mathrm{ex}} \ll \epsilon^{-2}$. 


\section{An upper bound construction via Ginzburg-Landau}

We begin by constructing a periodic two-dimensional configuration which gives a good highest-order approximation to the Ginzburg-Landau energy,

$$
G_{\epsilon}(v, A)=\int_{Q}\left\{\frac{1}{2}|(\nabla-i A) v|^{2}+\frac{1}{4 \epsilon^{2}}\left(|v|^{2}-1\right)^{2}+\frac{1}{2}\left(\nabla \times A-h_{\mathrm{ex}}\right)^{2}\right\} d \vec{x},
$$

for $v \in H_{\text {loc }}^{1}\left(\mathbb{R}^{2} ; \mathbb{C}\right), A \in H_{\text {loc }}^{1}\left(\mathbb{R}^{2} ; \mathbb{R}^{2}\right)$ satisfying two-dimensional Floquet boundary conditions with respect to the unit square $Q=[0,1]^{2}$,

$$
\left\{\begin{array}{l}
v\left(\vec{x}+\vec{e}_{j}\right)=v(\vec{x}) e^{i \omega_{j}(\vec{x})}, \quad j=1,2, \\
A\left(\vec{x}+\vec{e}_{j}\right)=A(\vec{x})+\nabla \omega_{j}(\vec{x}), \quad j=1,2,
\end{array}\right.
$$

for $\omega_{j} \in H_{\mathrm{loc}}^{2}\left(\mathbb{R}^{2}\right), j=1,2$. The choice of period domain $Q=[0,1]^{2}$ is made for simplicity, and any other parallelogram may be substituted with identical result for $|Q|^{-1} G_{\epsilon}$. We refer to Proposition 8.1 in [SS] for the modification needed to handle a general domain $\Omega$.

Proposition 4.2. Assume that $\epsilon>0$ and $h_{\mathrm{ex}} \ll \epsilon^{-2}$.

1. If $h_{\mathrm{ex}}=H_{\mathrm{ex}}|\ln \epsilon|$ for constant $H_{\mathrm{ex}}>0$, then for any given constant $H \in \mathbb{R}$ there exists a configuration $\left(u_{\epsilon}, A_{\epsilon}\right)$ satisfying (4.2) such that

$$
G_{\epsilon}\left(u_{\epsilon}, A_{\epsilon}\right) \leq \frac{1}{2}\left[|H|+\left(H-H_{\mathrm{ex}}\right)^{2}\right]|\ln \epsilon|^{2}+o\left(|\ln \epsilon|^{2}\right) .
$$

2. If $|\ln \epsilon| \ll h_{\mathrm{ex}} \ll \epsilon^{-2}$, then there exists a configuration $\left(u_{\epsilon}, A_{\epsilon}\right)$ satisfying (4.2) such that

$$
G_{\epsilon}\left(u_{\epsilon}, A_{\epsilon}\right) \leq \frac{1}{2} h_{\mathrm{ex}} \ln \left(\frac{1}{\epsilon \sqrt{h_{\mathrm{ex}}}}\right)+O\left(h_{\mathrm{ex}}\right) .
$$

Proof. Let $K=[-1 / 2,1 / 2]^{2}$, and consider the solution of the boundary-value problem

$$
\left\{\begin{array}{l}
-\Delta f=2 \pi\left(\delta_{0}-1\right) \quad \text { in } K, \\
\partial_{\nu} f=0 \text { on } \partial K \\
\int_{K} f=0 .
\end{array}\right.
$$

By standard elliptic theory the solution exists and is unique, and $f(x)+\ln |x|$ is smooth in $K \backslash\{0\}$, where we let $x=\vec{x}$. As a result we have

$$
\int_{K \backslash B_{r}(0)} \frac{1}{2}|\nabla f|^{2} d x=\pi \ln \left(\frac{1}{r}\right)+O(1)
$$

as $r \rightarrow 0$. Note that $f$ is even in both directions, and hence it may be extended periodically to all $\mathbb{R}^{2}$. 
We now construct our configuration. First, we choose a potential $B$ with $\nabla \times B=2 \pi$. To fix ideas, let $B\left(x_{1}, x_{2}\right)=\pi\left(-x_{2}, x_{1}\right)$. Then define $v=e^{i \phi_{1}}$ in $\mathbb{R}^{2} \backslash \mathbb{Z}^{2}$ by

$$
\nabla \phi_{1}=\nabla^{\perp} f+B
$$

This is well-defined, since for any closed curve $\gamma \subset \mathbb{R}^{2} \backslash \mathbb{Z}^{2}, \gamma=\partial \omega$,

$$
\int_{\gamma}\left(\nabla^{\perp} f+B\right) \cdot d \vec{s}=\int_{\omega} \nabla \times\left(\nabla^{\perp} f+B\right)=\int_{\omega}(-\Delta f+2 \pi) \in 2 \pi \mathbb{Z} .
$$

Now we rescale and truncate $(v, B)$ to create a test configuration with the correct leading order energy. Choose a smooth cut-off $\rho_{\eta} \in C^{\infty}(K)$,

$$
\rho_{\eta}(x)= \begin{cases}0, & |x| \leq \eta \\ 1, & |x| \geq 2 \eta\end{cases}
$$

and extend $\rho_{\eta}$ to $\mathbb{R}^{2}$ periodically. Then, for $0<\epsilon<\delta$ define

$$
u_{\epsilon, \delta}(x)=\left[\rho_{\epsilon / \delta} v\right](x / \delta), \quad A_{\delta}(x)=\delta^{-1} B(x / \delta) .
$$

In order that $\left(u_{\epsilon, \delta}, A_{\delta}\right)$ be gauge periodic with respect to $Q$ we require that $\delta^{-1} \in \mathbb{Z}$, a condition which will be met by approximation (since we will always take $h_{\mathrm{ex}} \rightarrow \infty$ ). Assuming this compatibility condition on $\delta$, we then calculate

$$
\left(\nabla-i A_{\delta}\right) u_{\epsilon, \delta}(x)=\delta^{-1}(\nabla-i B)\left[\rho_{\epsilon / \delta} v\right](y),
$$

where $y=x / \delta$, and

$$
\nabla \times A_{\delta}(x)=\delta^{-2} \nabla \times B(y)=2 \pi \delta^{-2} .
$$

We then have

$$
\begin{aligned}
\int_{Q}\left|\nabla \times A_{\delta}-h_{\mathrm{ex}}\right|^{2} & =\delta^{-2} \int_{Q_{1 / \delta}}\left|\nabla \times B-\delta^{2} h_{\mathrm{ex}}\right|^{2}=\delta^{-4} \int_{K}\left|\nabla \times B-\delta^{2} h_{\mathrm{ex}}\right|^{2} \\
& =\left|2 \pi \delta^{-2}-h_{\mathrm{ex}}\right|^{2}
\end{aligned}
$$

where $Q_{1 / \delta}=[0,1 / \delta]^{2}$.

Next, we have

$$
\begin{aligned}
\int_{Q}[ & \left.\frac{1}{2}\left|\left(\nabla-i A_{\delta}\right) u_{\epsilon, \delta}\right|^{2}+\frac{1}{4 \epsilon^{2}}\left(\left|u_{\epsilon, \delta}\right|^{2}-1\right)^{2}\right] \\
& =\delta^{-2} \int_{Q_{\delta}}\left[\frac{1}{2} \mid\left(\nabla-i A_{\delta}\right) u_{\epsilon, \delta}^{2}+\frac{1}{4 \epsilon^{2}}\left(\left|u_{\epsilon, \delta}\right|^{2}-1\right)^{2}\right] \\
& =\delta^{-2} \int_{K}\left[\frac{1}{2 \delta^{2}}\left|(\nabla-i B)\left(\rho_{\epsilon / \delta} v\right)\right|^{2}+\frac{1}{4 \epsilon^{2}}\left(\left|\rho_{\epsilon / \delta}\right|^{2}-1\right)^{2}\right] \delta^{2} d y \\
& \leq \delta^{-2} \int_{K \backslash B_{\epsilon / \delta}} \frac{1}{2}\left|\nabla \phi_{1}-B\right|^{2}+\delta^{-2} \int_{B_{2 \epsilon / \delta} \backslash B_{\epsilon / \delta}}\left[\frac{1}{2}\left|\nabla \rho_{\epsilon / \delta}\right|^{2}+\frac{\delta^{2}}{4 \epsilon^{2}}\left(\rho_{\epsilon / \delta}^{2}-1\right)^{2}\right] \\
& \leq \delta^{-2} \int_{K \backslash B_{\epsilon / \delta}} \frac{1}{2}|\nabla f|^{2}+O\left(\delta^{-2}\right)=\pi \delta^{-2} \ln (\delta / \epsilon)+O\left(\delta^{-2}\right) .
\end{aligned}
$$


Putting the above estimate and (4.6) together we obtain the upper bound

$$
G_{\epsilon}\left(u_{\epsilon, \delta}, A_{\delta}\right) \leq \pi \delta^{-2} \ln (\delta / \epsilon)+\frac{1}{2}\left|2 \pi \delta^{-2}-h_{\mathrm{ex}}\right|^{2}+O\left(\delta^{-2}\right) .
$$

To conclude we must choose $\delta$ appropriately for each case. In case $h_{\mathrm{ex}}=H_{\mathrm{ex}}|\ln \epsilon|$, given any constant $H$, we choose $\delta$ so that $\delta^{-1} \in \mathbb{Z}$ is the closest integer to $\sqrt{H|\ln \epsilon| / 2 \pi}$. In particular, we then have $\left|2 \pi \delta^{-2}-h_{\mathrm{ex}}\right|=|H| \ln \epsilon\left|-h_{\mathrm{ex}}\right|+O(1 / \delta)$, and hence the upper bound (4.7) implies

$$
G_{\epsilon}(u, A) \leq \frac{1}{2}\left[|H|+\left(H-H_{\mathrm{ex}}\right)^{2}\right]|\ln \epsilon|^{2}+O(|\ln \epsilon| \ln |\ln \epsilon|) .
$$

In case $h_{\mathrm{ex}} \gg|\ln \epsilon|$, we instead make $\delta^{-1} \in \mathbb{Z}$ the closest integer to $\sqrt{h_{\mathrm{ex}} / 2 \pi}$. In that case

$$
\int_{Q}\left|\nabla \times A_{\delta}-h_{\mathrm{ex}}\right|^{2}=O(1)
$$

and (4.7) now yields

$$
G_{\epsilon}(u, A) \leq \frac{1}{2} h_{\mathrm{ex}} \ln \frac{1}{\epsilon \sqrt{h_{\mathrm{ex}}}}+O\left(h_{\mathrm{ex}}\right)
$$

We plan to obtain the upper bound for the Lawrence-Doniach energy using the configuration constructed above, with Ginzburg-Landau parameter $\eta=s / \sigma$ (with $\sigma=\sigma(s)$ to be chosen below, giving a periodic vortex lattice with lattice spacing $O(\delta)=O\left(h_{\mathrm{ex}}^{-1 / 2}\right)$ and vortices of core size $O(\eta)$. In principle, the minimizer of the Lawrence-Doniach functional should roughly resemble this configuration, but with $u_{\eta}$ discretized to take values only on the superconducting planes. Here is where the details of the model play a subtle role. Indeed, if a row of vortices lies across a plane $P_{n}$, the contribution to the LawrenceDoniach energy will be much larger than desired, on the order of $s^{2} / \sigma^{2} \epsilon^{2}$ per vortex. A much smaller energy will be obtained by fitting the rows of vortices between adjacent planes, so that the order parameter $u_{\eta}$ never vanishes on the planes $P_{n}$. Unfortunately, the intervortex distance $\delta$ may not be an exact multiple of the interplanar distance $s$, and so we will need to make a small perturbation in the periodic vortex lattice so as to locate the vortices between the planes where they normally should be observed.

Lemma 4.3. Let $\eta>0, h_{\mathrm{ex}}$ be as in Proposition 4.2, and assume $\eta \lesssim s$. There exists a configuration $\left(\tilde{u}_{s, \delta}, \tilde{A}_{s, \delta}\right)$ such that the set

$$
Z_{s, \eta, \delta}=\left\{z:\left|\tilde{u}_{s, \delta}(x, z)\right|<1 \text { for some } x \in[0,1]\right\}
$$

consists of $\delta^{-1}$ strips and is contained in $\bigcup_{n=1}^{N}[(n+1 / 2) s-\eta,(n+1 / 2) s+\eta]$, and

$$
G_{\eta}\left(\tilde{u}_{s, \delta}, \tilde{A}_{s, \delta}\right) \leq \begin{cases}\frac{1}{2}\left[|H|+\left(H-H_{\mathrm{ex}}\right)^{2}\right]|\ln \eta|^{2}+o\left(|\ln \eta|^{2}\right) & \text { if } h_{\mathrm{ex}} \leq H_{\mathrm{ex}}|\ln \eta|, \\ \frac{1}{2} h_{\mathrm{ex}} \ln \left(\frac{1}{\eta \sqrt{h_{\mathrm{ex}}}}\right)[1+o(1)] & \text { if } \ln \eta \ll h_{\mathrm{ex}} \ll \eta^{-2} .\end{cases}
$$


Proof. We return to the construction in the proof of Proposition 4.2. Choose two bands,

$$
E^{+}=[-1 / 2,1 / 2] \times[1 / 8,3 / 8], E^{-}=[-1 / 2,1 / 2] \times[-3 / 8,-1 / 8] \subset Q .
$$

Since $f(x)$ (defined in (4.4)) is smooth in $E^{ \pm}$, we may conclude $|\nabla f(x)| \leq C$ for $x \in E^{ \pm}$, with constant $C$. Following the construction in the proof we replicate $E^{+} \cup E^{-}$ periodically to form a series of horizontal strips in $\mathbb{R}^{2}$, and rescale by $\delta^{-1}$ to obtain a set of horizontal strips of width $\delta / 4$. Restricting to the basic period domain $Q$, we obtain a set $E_{\delta}$ consisting of $2 \delta^{-1}$ horizontal strips, each of width $\delta / 4$,

$$
\begin{gathered}
E_{\delta}=\bigcup_{m=1}^{\delta^{-1}}\left(E_{\delta, m}^{-} \cup E_{\delta, m}^{+}\right), \\
E_{\delta, m}^{-}=\left\{(x, z) \in Q: \zeta_{m}-3 \delta / 8 \leq z \leq \zeta_{m}-\delta / 8\right\}, \\
E_{\delta, m}^{+}=\left\{(x, z) \in Q: \zeta_{m}+\delta / 8 \leq z \leq \zeta_{m}+3 \delta / 8\right\},
\end{gathered}
$$

where $\zeta_{m}=(m+1 / 2) \delta, m=1, \ldots, \delta^{-1}$, the $z$-coordinate of the vortex locations in the construction of $u_{\eta}$. Using the scaling and cut-off (4.5) we conclude that

$$
\left|\left(\nabla-i A_{\eta}\right) u_{\eta}\right| \leq C \delta^{-1}
$$

for all $(x, z) \in E_{\delta}$.

We now describe how to move the row of vortices located at $z=\zeta_{m}$ so that they be located exactly at the midpoint between adjacent pairs of planes. For each $m$, we identify the planes which lie around the vortex row: choose $n$ such that $z_{n-1}<\zeta_{m} \leq z_{n}$. If $\zeta_{m}$ lies below (respectively, above) the midpoint between the planes $z=z_{n-1}$ and $z=z_{n}$, we slightly expand (resp., contract) the strip $E_{\delta, m}^{-}$below the row, and slightly contract (resp., expand) the strip $E_{\delta, m}^{+}$above the row, so as to move the row $\zeta_{m}$ to the midpoint $z=\frac{1}{2}\left(z_{n-1}+z_{n}\right)$. Since the distance between the rows is $\delta \gg s$, the distortion is small, and the change in energy will be neglibible.

Following this plan, for each $m$ we choose $t_{m} \in(-s / 2, s / 2]$ so that $\zeta_{m}+t_{m}=$ $(n+1 / 2) s$. Define a piecewise linear transformation $\Phi_{s, \delta}^{m}(x, z)$ which maps each strip $\left\{\zeta_{m}-\delta / 2 \leq z \leq \zeta_{m}+\delta / 2\right\}$ to itself, as follows:

$$
\Phi_{s, \delta}^{m}(x, z)= \begin{cases}(x, z) & \text { if } \zeta_{m}-\delta / 2 \leq z \leq \zeta_{m}-\delta / 4-s \\ \left(x, z+t_{m}\right) & \text { if } \zeta_{m}-\delta / 4+s \leq z \leq \zeta_{m}+\delta / 4-s \\ (x, z) & \text { if } \zeta_{m}+\delta / 4+s \leq z \leq \zeta_{m}+\delta / 2\end{cases}
$$

and $\Phi_{s, \delta}^{m}$ is linear (in $z$, identity in $x$ ) in the transition regions. Next, define $\Phi_{s, \delta}: Q \rightarrow Q$ to agree with $\Phi_{s, \delta}^{m}$ in each horizontal strip of height $\delta$. In this way, $\Phi_{s, \delta}$ is piecewise $C^{1}$, and since it is the identity along the top edges of $Q$ (and independent of $x$ throughout) it extends periodically to $\mathbb{R}^{2}$. Now, let $\Psi_{s, \delta}=\Phi_{s, \delta}^{-1}$. By the construction, we note that $\Phi_{s, \delta}$ is invertible, and both $\left|D \Phi_{s, \delta}\right|=\left|\operatorname{det} D \Phi_{s, \delta}\right| \leq 2,\left|D \Psi_{s, \delta}\right|=\left|\operatorname{det} D \Psi_{s, \delta}\right| \leq 2$.

We then define our modified configuration, $\left(\tilde{u}_{s, \delta}, \tilde{A}_{s, \delta}\right)$,

$$
\tilde{u}_{s, \delta}(x, z)=u_{\eta}\left(\Psi_{s, \delta}(x, z)\right), \quad \tilde{A}_{s, \delta}=\Psi_{s, \delta}^{*} A_{\eta},
$$


where we treat $A_{\eta}$ as a 1 -form. Since $\Psi_{s, \delta}$ is a rigid motion except for the $2 \delta^{-1}$ strips of width $2 s$ (where the linear deformation takes place), the energy of $\left(\tilde{u}_{s, \delta}, \tilde{A}_{s, \delta}\right)$ is unchanged except for these strips. These transition strips are contained within the set $E_{\delta}$ above, and therefore the energy density in these strips is uniformly bounded by $C \delta^{-2}$, by (4.8). Thus, the difference in energy satisfies

$$
\left|G_{\eta}\left(u_{\eta}, A_{\eta}\right)-G_{\eta}\left(\tilde{u}_{s, \delta}, \tilde{A}_{s, \delta}\right)\right| \leq 2 s \cdot 2 \delta^{-1} \cdot C \delta^{-2}=C^{\prime} s \sqrt{h_{\mathrm{ex}}} h_{\mathrm{ex}}=o(1) h_{\mathrm{ex}} .
$$

Thus, the claim is established.

We now prove our upper bound on the Lawrence-Doniach energy:

Proposition 4.4. For any $H \in \mathbb{R}$, there exists $\left(v_{s, n}, B_{s}\right)$ satisfying (1.1) with

$$
\frac{1}{|Q|} \mathcal{L}_{\epsilon, s}\left(v_{s, n}, B_{s}\right) \leq \begin{cases}\frac{1}{2}\left[|H|+\left(H-H_{\mathrm{ex}}\right)^{2}\right]|\ln s|^{2}+o\left(|\ln s|^{2}\right) & \text { if } h_{\mathrm{ex}} \leq H_{\mathrm{ex}}|\ln s|, \\ \frac{1}{2} h_{\mathrm{ex}} \ln \left(\frac{1}{s \sqrt{h_{\mathrm{ex}}}}\right)[1+o(1)] & \text { if } \ln s \ll h_{\mathrm{ex}} \ll s^{-2} .\end{cases}
$$

Proof. Define $\sigma$ so that $1 \ll \ln \sigma \ll \ln \frac{1}{s \sqrt{h_{\mathrm{ex}}}}$, which is possible under the hypothesis $s^{2} h_{\mathrm{ex}} \ll 1$. Then, we apply Lemma 4.3 with $\eta=s / \sigma$ to get $(u, A)$ satisfying the bounds

$$
\begin{aligned}
G_{\eta}(u, A) & \leq \begin{cases}\frac{1}{2}\left[|H|+\left(H-H_{\mathrm{ex}}\right)^{2}\right]\left|\ln \frac{s}{\sigma}\right|^{2}+o\left(\left|\ln \frac{s}{\sigma}\right|^{2}\right) & \text { if } h_{\mathrm{ex}} \leq H_{\mathrm{ex}}\left|\ln \frac{s}{\sigma}\right|, \\
\frac{1}{2} h_{\mathrm{ex}} \ln \left(\frac{\sigma}{s \sqrt{h_{\mathrm{ex}}}}\right)[1+o(1)] & \text { if } \ln \frac{s}{\sigma} \ll h_{\mathrm{ex}} \ll \frac{\sigma^{2}}{s^{2}},\end{cases} \\
& \leq \begin{cases}\frac{1}{2}\left[|H|+\left(H-H_{\mathrm{ex}}\right)^{2}\right]|\ln s|^{2}+o\left(|\ln s|^{2}\right) & \text { if } h_{\mathrm{ex}} \leq H_{\mathrm{ex}}|\ln s|, \\
\frac{1}{2} h_{\mathrm{ex}} \ln \left(\frac{1}{s \sqrt{h_{\mathrm{ex}}}}\right)[1+o(1)] & \text { if } \ln s \ll h_{\mathrm{ex}} \ll s^{-2},\end{cases}
\end{aligned}
$$

and whose vortices lie in the set $\bigcup_{n=1}^{N}[(n+1 / 2) s-\eta,(n+1 / 2) s+\eta]$.

The general idea is to use the mean-value theorem and periodicity to choose a vertical translation of $(u, A)$ which when restricted to the planes $P_{n}$ gives the same energy for Lawrence-Doniach. However, we must exclude translations which move the rows of vortices to within distance $\eta$ of planes $P_{n}$. We define the set of "good" translations,

$$
\begin{aligned}
\mathcal{T} & =\left\{t \in[-s / 2, s / 2):|u|=1 \text { for all }\left(x, z_{n}\right) \in P_{n} \text { and } n=1, \ldots, N\right\} \\
& =[-s / 2+s / \sigma, s / 2-s / \sigma),
\end{aligned}
$$

for which none of the vortex balls cross the planes. Having chosen $\sigma \gg 1$, the excluded translations are very small compared with the layer spacing $s$, and will not contribute to the energy estimate we obtain.

Applying Fubini's theorem with

$$
f=\left|\left(\partial_{x}-i A_{x}\right) u\right|^{2}+\frac{\sigma^{2}}{2 s^{2}}\left(1-|u|^{2}\right)^{2},
$$


we observe that there exists $t_{*} \in \mathcal{T}$ so that

$$
\begin{aligned}
\int_{Q} f(x, z) d x d z & =\int_{t=-s / 2}^{s / 2}\left(\sum_{n=1}^{N} \int_{0}^{1} f\left(x, z_{n}+t\right) d x\right) d t \\
& \geq \int_{\mathcal{T}}\left(\sum_{n=1}^{N} \int_{0}^{1} f\left(x, z_{n}+t\right) d x\right) d t \\
& \geq|\mathcal{T}| \sum_{n=1}^{N} \int_{0}^{1} f\left(x, z_{n}+t_{*}\right) d x \geq\left(1-\sigma^{-1}\right) s \sum_{n=1}^{N} \int_{0}^{1} f\left(x, z_{n}+t_{*}\right) d x .
\end{aligned}
$$

Define $v_{s, n}(x)=u\left(x, z_{n}+t_{*}\right)$ and $B_{S}(x, z)=A\left(x, z+t_{*}\right)$. Since $t_{*} \in \mathcal{T}$, it follows that $\left|v_{s, n}(x)\right|=1$ for all $n, x$, and hence

$$
\begin{aligned}
s \sum_{n=1}^{N} \int_{0}^{1}\left[\mid\left(\partial_{x}-\right.\right. & \left.\left.i B_{s, x}\right)\left.v_{s, n}\right|^{2}+\frac{1}{2 \epsilon^{2}}\left(1-\left|v_{s, n}\right|^{2}\right)^{2}\right] d x \\
& =s \sum_{n=1}^{N} \int_{0}^{1}\left[\left|\left(\partial_{x}-i B_{s, x}\right) v_{s, n}\right|^{2}+\frac{\sigma^{2}}{2 s^{2}}\left(1-\left|v_{s, n}\right|^{2}\right)^{2}\right] d x \\
& \leq(1+o(1)) \int_{Q}\left[\left|\left(\partial_{x}-i A_{x}\right) u\right|^{2}+\frac{\sigma^{2}}{2 s^{2}}\left(1-|u|^{2}\right)^{2}\right] d x d z .
\end{aligned}
$$

On the other hand, dropping the subscript $\epsilon$,

$$
\begin{aligned}
\frac{v_{n}-v_{n-1} e^{i \int_{z_{n-1}}^{z_{n}} B_{s, z}\left(x, z^{\prime}\right) d z^{\prime}}}{s} & =\frac{1}{s} \int_{z_{n-1}+t}^{z_{n}+t} \partial_{z}\left(u(x, z) e^{i \int_{z}^{z n+t} A_{z}\left(x, z^{\prime}\right) d z^{\prime}}\right) d z \\
& =\frac{1}{s} \int_{z_{n-1}+t}^{z_{n}+t}\left(\partial_{z} u-i A_{z} u\right) e^{i \int_{z}^{z_{n}+t} A_{z}\left(x, z^{\prime}\right) d z^{\prime}} d z .
\end{aligned}
$$

Thus, using Cauchy-Schwarz and summing,

$$
\sum_{n=1}^{N} \int_{0}^{1}\left\{\frac{1}{s^{2}}\left|v_{n}-v_{n-1} e^{i \int_{z_{n-1}}^{z_{n}} B_{s, z}\left(x, z^{\prime}\right) d z^{\prime}}\right|^{2}\right\} d x \leq \int_{Q}\left|\left(\partial_{z}-i A_{z}\right) u\right|^{2} d x d z .
$$

From (4.9) and (4.10) we deduce that $\mathcal{L}_{\epsilon, s}\left(v_{s, n}, B_{s}\right) \leq G_{\eta}(u, A)(1+o(1))$.

\section{Lower bounds}

We will use the tools developed in [ABS1] to derive lower bounds for the energy in this regime. Let $\left(u_{n}, A\right)$ be minimizers of $\mathcal{L}_{\epsilon, s}$. We introduce an interpolated order parameter by letting, for $z \in\left(z_{n-1}, z_{n}\right)$,

$$
\Psi(x, z):=\left[\frac{z-z_{n-1}}{s} u_{n}(x)+\frac{z_{n}-z}{s} u_{n-1}(x) e^{i \int_{z_{n-1}}^{z_{n}} A_{z}\left(x, z^{\prime}\right) d z^{\prime}}\right] e^{-i \int_{z}^{z_{n}} A_{z}\left(x, z^{\prime}\right) d z^{\prime}} .
$$


We again note that $\Psi$ satisfies the Floquet boundary conditions (2.5) (now with $j=1,2$ only).

As in [ABS1], we have the following comparison bounds between terms in $\mathcal{L}_{\epsilon, s}$ and $G_{S}$ :

Lemma 4.5. Assume $\left(u_{n}, A\right)$ are minimizers of $\mathcal{L}_{\epsilon, s}$, and $\Psi$ is defined as in (4.11). Then

$$
\begin{aligned}
& \int_{Q}|(\nabla-i \vec{A}) \Psi|^{2} d x d z \\
& \quad \leq s \sum_{n=1}^{N} \int_{0}^{1}\left\{\left|\left(\partial_{x}-i A_{x}\left(x, z_{n}\right)\right) u_{n}\right|^{2}+\frac{1}{s^{2}}\left|u_{n}-u_{n-1} e^{i \int_{z_{n-1}}^{z_{n}} A_{z}\left(x, z^{\prime}\right) d z^{\prime}}\right|^{2}\right\} d x \\
& \quad+o\left(\mathcal{L}_{\epsilon, s}\left(u_{n}, A\right)\right) \\
& \int_{Q} \frac{1}{s^{2}}\left(1-|\Psi|^{2}\right)^{2} d x d z \\
& \leq s \sum_{n=1}^{N}\left[\frac{2}{s^{2}} \int_{0}^{1}\left(1-\left|u_{n}\right|^{2}\right)^{2} d x+\frac{1}{2 s^{2}} \int_{0}^{1}\left|u_{n}-u_{n-1} e^{i \int_{z_{n-1}}^{z_{n}} A_{z}\left(x, z^{\prime}\right) d z^{\prime}}\right|^{2} d x\right] .
\end{aligned}
$$

Proof. Many of the computations are unchanged from [ABS1]. Estimate (4.13) follows exactly as in Lemma 4.2 of [ABS1]. An explicit calculation shows

$$
\left(\partial_{z}-i A_{z}(x, z)\right) \Psi(x, z)=\frac{1}{s}\left[u_{n}-u_{n-1} e^{i \int_{z_{n-1}}^{z_{n}} A_{z}\left(x, z^{\prime}\right) d z^{\prime}}\right] .
$$

It remains to estimate the $x$-derivative term. Here we use an observation which is specific to the parallel case, in which the problem is two-dimensional. The Euler-Lagrange equations for $\mathcal{L}_{\epsilon, s}$ in this case (see [ABS1]) imply that the magnetic field $h(x, z)=h_{y}(x, z)=$ $\partial_{z} A_{x}-\partial_{x} A_{z}$ is constant in $z$ in each of the gaps between the planes:

$$
h(x, z)=h^{(n)}(x), \quad z_{n-1}<z<z_{n} .
$$

With this simplification, and letting $t=\left(z-z_{n-1}\right) / s$, we may calculate

$$
\begin{aligned}
&\left|\left(\partial_{x}-i A_{x}(x, z)\right) \Psi\right|^{2} \\
&=\mid t\left(\partial_{x}-i A_{x}\left(x, z_{n}\right)\right) u_{n}+(1-t) e^{i \int_{z_{n-1}}^{z_{n}} \tilde{A}_{z}\left(x, z^{\prime}\right) d z^{\prime}}\left(\partial_{x}-i A_{x}\left(x, z_{n-1}\right)\right) u_{n-1} \\
& \quad-\left.i s t(1-t) h^{(n)}(x)\left[u_{n}-u_{n-1} e^{i \int_{z_{n-1}}^{z_{n}} A_{z}\left(x, z^{\prime}\right) d z^{\prime}}\right]\right|^{2} \\
& \leq(1+\gamma)\left|t\left(\partial_{x}-i A_{x}\left(x, z_{n}\right)\right) u_{n}+(1-t) e^{i \int_{z_{n-1}}^{z_{n}} A_{z}\left(x, z^{\prime}\right) d z^{\prime}}\left(\partial_{x}-i A_{x}\left(x, z_{n-1}\right)\right) u_{n-1}\right|^{2} \\
&+\left(1+\gamma^{-1}\right) \mid s t(1-t) h^{(n)}(x)\left[u_{n}-\left.u_{n-1} e^{i \int_{z_{n-1}}^{z_{n}} A_{z}\left(x, z^{\prime}\right) d z^{\prime}}\right|^{2}\right. \\
& \leq(1+\gamma)\left\{t\left|\left(\partial_{x}-i A_{x}\left(x, z_{n}\right)\right) u_{n}\right|^{2}+(1-t)\left|\left(\partial_{x}-i A_{x}\left(x, z_{n-1}\right)\right) u_{n-1}\right|^{2}\right\} \\
&+\left(1+\gamma^{-1}\right) \frac{1}{16} s^{2}\left(h^{(n)}(x)\right)^{2}\left|u_{n}-u_{n-1} e^{i \int_{z_{n-1}}^{z_{n}} A_{z}\left(x, z^{\prime}\right) d z^{\prime}}\right|^{2}
\end{aligned}
$$


with $\gamma>0$ (to be determined), and where we have used Jensen's inequality to estimate the square of the convex combination in the last line. We now choose

$$
\gamma=s^{2} h_{\mathrm{ex}} \ll 1
$$

and show that, with this choice, the last line is negligible. We use $\left(h^{(n)}\right)^{2} \leq 2 h_{\mathrm{ex}}^{2}+$ $2\left(h^{(n)}-h_{\mathrm{ex}}\right)^{2}$ and estimate the last line for each summand. First, we note

$$
\begin{aligned}
s \sum_{n=1}^{N} \int_{Q_{n}}\left(1+\gamma^{-1}\right) & s^{2} h_{\mathrm{ex}}^{2}\left|u_{n}-u_{n-1} e^{i \int_{z_{n-1}}^{z_{n}} A_{z}\left(x, z^{\prime}\right) d z^{\prime}}\right|^{2} \\
& \leq s \sum_{n=1}^{N} \int_{Q_{n}}\left(1+\gamma^{-1}\right)\left(s^{2} h_{\mathrm{ex}}\right)^{2} \frac{1}{s^{2}}\left|u_{n}-u_{n-1} e^{i \int_{z_{n-1}}^{z_{n}} A_{z}\left(x, z^{\prime}\right) d z^{\prime}}\right|^{2} \\
& \leq 2\left(s^{2} h_{\mathrm{ex}}\right) s \sum_{n=1}^{N} \int_{Q_{n}} \frac{1}{s^{2}}\left|u_{n}-u_{n-1} e^{i \int_{z_{n-1}}^{z n} A_{z}\left(x, z^{\prime}\right) d z^{\prime}}\right|^{2} \\
& \leq 2 s^{2} h_{\mathrm{ex}} C h_{\mathrm{ex}} \ln \frac{1}{s^{2} h_{\mathrm{ex}}}=o\left(\mathcal{L}_{\epsilon, s}\left(u_{n}, A\right)\right)
\end{aligned}
$$

which is indeed a term of smaller order of energy. Finally, we calculate

$$
\begin{aligned}
s \sum_{n=1}^{N} \int_{Q_{n}}\left(1+\gamma^{-1}\right) s^{2}\left(h^{(n)}-\right. & \left.h_{\mathrm{ex}}\right)^{2}\left|u_{n}-u_{n-1} e^{i \int_{z_{n-1}}^{z_{n}} A_{z}\left(x, z^{\prime}\right) d z^{\prime}}\right|^{2} \\
& \leq s \sum_{n=1}^{N} \int_{Q_{n}}\left(1+\gamma^{-1}\right) 4 s^{2}\left|h(x, z)-h_{\mathrm{ex}}\right|^{2} \\
& \leq 8\left(s^{2} h_{\mathrm{ex}}\right)^{-1} s^{2} \int_{Q}\left|h-h_{\mathrm{ex}}\right|^{2} \leq \frac{4}{h_{\mathrm{ex}}} C h_{\mathrm{ex}} \ln \frac{1}{s^{2} h_{\mathrm{ex}}}
\end{aligned}
$$

Putting these two estimates together, we integrate (4.14) to obtain

$$
\begin{array}{rl}
\int_{Q}\left|\left(\partial_{x}-i A_{x}(x, z)\right) \Psi\right|^{2} \\
\leq s & s \sum_{n=1}^{N} \int_{P_{n}}(1+\gamma)\left\{t\left|\left(\partial_{x}-i A_{x}\left(x, z_{n}\right)\right) u_{n}\right|^{2}+(1-t)\left|\left(\partial_{x}-i A_{x}\left(x, z_{n-1}\right)\right) u_{n-1}\right|^{2}\right\} \\
& +o\left(C h_{\mathrm{ex}} \ln \frac{1}{s^{2} h_{\mathrm{ex}}}\right) \\
& \leq s \sum_{n=1}^{N} \int_{P_{n}}\left|\left(\partial_{x}-i A_{x}\left(x, z_{n}\right)\right) u_{n}\right|^{2}+o\left(h_{\mathrm{ex}} \ln \frac{1}{s^{2} h_{\mathrm{ex}}}\right)
\end{array}
$$

using the periodicity in $n$ and the definition of $\gamma=s^{2} h_{\mathrm{ex}} \ll 1$. This proves (4.12). 
As the Lawrence-Doniach energy is roughly but not exactly bounded below by the Ginzburg-Landau energy of the interpolation $\Psi$, we need to introduce a modified functional as in $[\mathrm{ABS} 1]$,

$\mathcal{M}_{s}(\Psi, A, Q)$

$=\frac{1}{2} \int_{Q}\left\{|\Psi|^{2}|(\nabla-i A) \Psi|^{2}+\frac{1}{2}\left(1-|\Psi|^{2}\right)\left(|\nabla| \Psi||^{2}+\frac{\alpha}{s^{2}}\left(1-|\Psi|^{2}\right)^{2}\right)+\left(h-h_{\mathrm{ex}}\right)^{2}\right\}$.

We recall that $\alpha$ comes from our hypothesis $\epsilon \leq s / \alpha$. From Lemma 4.4 of [ABS1] we have, for energy minimizers $\left(u_{n}, A\right)$,

$$
\mathcal{L}_{\epsilon, s}\left(u_{n}, A\right) \geq \mathcal{M}_{s}(\Psi, A)+o\left(h_{\mathrm{ex}} \ln \frac{1}{s^{2} h_{\mathrm{ex}}}\right) .
$$

When $h_{\mathrm{ex}} \leq C|\ln s|^{k} \ll s^{-1}$ for some $k>0$, we may derive a lower bound on the energy via the ball-construction method [Sa], [Je]:

Proposition 4.6. Choose any $k, l>0$. Assume that $h_{\mathrm{ex}} \leq C|\ln s|^{k}, \mathcal{L}_{\epsilon, s}\left(u_{n}, A\right) \leq$ $C|\ln s|^{k}$ and $\Psi$ is defined as in (4.11). Then, for any small enough $s$, there exists a $f$ nite collection $\mathcal{B}=\left\{B_{i}\right\}_{i \in I}$ of disjoint closed balls such that:

1. $r(\mathcal{B})=|\ln s|^{-l}$, where $r(\mathcal{B})$ denotes the sum of the radii of the balls in $\mathcal{B}$.

2. $\left\{x \in \Omega:|1-| \Psi|| \geq|\ln s|^{-l}\right\} \subset \bigcup_{i \in I} B_{i}$.

3. With $d_{i}=\operatorname{deg}\left(\Psi, \partial B_{i}\right), i \in I$, there is a constant $C$ such that

$$
\mathcal{M}_{s}\left(\Psi, A, B_{i}\right) \geq \pi d_{i}(|\ln s|-C \ln |\ln s|) .
$$

Proof. From the hypotheses and the lower bounds in Lemma 4.5 we immediately deduce the following bound for the classical Ginzburg-Landau energy (but with parameter $s$ !):

$$
G_{S}(\Psi, A) \leq 4 \mathcal{L}_{\epsilon, s}\left(u_{n}, A\right) \leq C|\ln s|^{k} .
$$

In particular (see for instance Proposition 4.8 of [SS]) there exists a collection of closed disjoint vortex balls, $\mathcal{B}_{0}=\left\{B_{i}^{0}\right\}_{i \in I}$, such that item 2 is satisfied and $r_{0}:=r\left(\mathcal{B}_{0}\right) \leq$ $C s|\ln s|^{m}$ with $m=k+2 l$. Then from Proposition 4.8 of [ABS1] there exists a collection $\mathcal{B}$ of balls of total radius $r=|\ln s|^{-l}$ ( $s$ being small enough so that $|\ln s|^{-l}>C s|\ln s|^{m}$ ) and such that the union of the balls in $\mathcal{B}$ contains the balls in $\mathcal{B}_{0}$. Moreover, if $B_{i} \in \mathcal{B}$ and $v=\Psi /|\Psi|$ then

$$
\int_{B_{i} \backslash \mathcal{B}_{0}}\left[|(\nabla-i \vec{A}) v|^{2}+(\operatorname{curl} \vec{A})^{2}\right] d x d y \geq 2 \pi\left|d_{i}\right|\left(\ln \frac{r}{r_{0}}-\frac{1}{2}\right),
$$

where $d_{i}$ is the degree of $v$ on $\partial B_{i}$. Using the fact that outside $\mathcal{B}_{0}$ we have $|\Psi| \geq$ $1-|\ln s|^{-l}$, we obtain

$$
|\Psi|^{2}|(\nabla-i A) \Psi|^{2} \geq\left(1-2|\ln s|^{-l}\right)|(\nabla-i \vec{A}) v|^{2}
$$

on $Q \backslash \mathcal{B}_{0}$. Together with (4.15) and replacing $r$ and $r_{0}$ by their values in (4.17) yields the desired result. 
Among other things, Proposition 4.6 gives a sense of what it means for the configuration $\left(u_{n}, A\right)$ to have vortices, since the discrete nature of the planes allows circulation of supercurrents along and between the planes without the vanishing of any of the $u_{n}$. As in the periodic Ginzburg-Landau model (see [ABS1, ABS2]) we may also use the Floquet boundary conditions to connect the total degree of vortices, $\sum_{i} d_{i}$, with the magnetic flux through the cross-section $Q$,

$$
\int_{Q} h=2 \pi \sum_{i} d_{i}
$$

Indeed, since there are only finitely many vortex balls for any fixed $s$, by translating the period square $Q$ if necessary, we may guarantee that none of the balls in $\mathcal{B}$ intersect $\partial Q$. Thus, $|\Psi| \geq 1 / 2$ on $\partial Q$ and the total degree may be calculated by integration of the phase of $\Phi=|\Phi| e^{i \phi}$ along $\partial Q$. Using the Floquet conditions (2.5), we observe that $\nabla \phi$ and $A$ satisfy the same relations with respect to the auxiliary functions $\omega_{1}, \omega_{2}$, and hence

$$
\sum_{i} d_{i}=\operatorname{deg}(\Psi, \partial Q)=\frac{1}{2 \pi} \int_{\partial Q} \nabla \phi \cdot \tau d s=\frac{1}{2 \pi} \int_{\partial Q} A \cdot \tau d s=\frac{1}{2 \pi} \int_{Q} h,
$$

by Stokes' Theorem. The claim is thus established.

We are now ready to give the lower bound for minimizers of the Lawrence-Doniach functional in case $h_{\mathrm{ex}}$ is of the order of $|\ln s|$ :

Proposition 4.7. Assume $h_{\mathrm{ex}}=H_{\mathrm{ex}}|\ln s|$ for constant $H$, and $\epsilon \leq s / \alpha$ for $\alpha>0$ constant. If $\left(u_{n}, A\right)$ are minimizers of $\mathcal{L}_{\epsilon, s}$ in $\mathcal{H}$, then for all $s>0$ sufficiently small,

$$
\frac{1}{|Q|} \mathcal{L}_{\epsilon, s}\left(u_{n}, A\right) \geq \frac{1}{2}\left|\int_{Q} h\right|+\frac{1}{2} \int_{Q}\left(h-h_{\mathrm{ex}}\right)^{2}+o\left(|\ln s|^{2}\right) .
$$

Proof. From (4.16) it suffices to prove the estimate for $\mathcal{M}_{s}(\Psi, A)$ with $\Psi$ given by (4.11). By Lemma 4.4 we may apply Proposition 4.6 with (say) $r=|\ln s|^{-10}$ to obtain for each small $s>0$ a family $\mathcal{B}=\left\{B_{i}\right\}$ of balls and degrees $d_{i}=\operatorname{deg}\left(\Psi, \partial B_{i}\right)$ such that the three conclusions of Proposition 4.6 hold. In particular, we have the lower estimate inside the balls,

$$
\begin{aligned}
\int_{\bigcup_{i} B_{i}}\left[\frac{1}{2}|\Psi|^{2}|\nabla \Psi|^{2}+r^{2} \mid \nabla\right. & \left.\times\left. A\right|^{2}+\frac{1}{2}\left(1-|\Psi|^{2}\right)\left(|\nabla| \Psi||^{2}+\frac{\alpha}{s^{2}}\left(1-|\Psi|^{2}\right)^{2}\right)\right] \\
& \geq \pi \sum_{i} d_{i}(|\ln s|-C \ln |\ln s|) \geq \frac{1}{2}\left|\int_{Q} h\right||\ln s|(1-o(1)),
\end{aligned}
$$

where we have used (4.18) to connect the total degree to the total magnetic flux through $Q$. Note that by the upper bound on the energy and the choice of $r=|\ln s|^{-10}$, we have

$$
r^{2} \int_{\bigcup_{i} B_{i}}(\nabla \times A)^{2} \leq 2 r^{2} \int_{Q}\left(\left(\nabla \times A-h_{\mathrm{ex}}\right)^{2}+h_{\mathrm{ex}}^{2}\right)=o(1) .
$$


Thus, we obtain

$$
\begin{gathered}
\mathcal{M}_{s}(\Psi, A) \\
\geq \int_{\bigcup_{i} B_{i}}\left[\frac{1}{2}|\Psi|^{2}|\nabla \Psi|^{2}+r^{2}|\nabla \times A|^{2}+\frac{1}{2}\left(1-|\Psi|^{2}\right)\left(\left.|\nabla| \Psi\right|^{2}+\frac{\alpha}{s^{2}}\left(1-|\Psi|^{2}\right)^{2}\right)\right. \\
\left.+\frac{1}{2}\left(\nabla \times A-h_{\mathrm{ex}}\right)^{2}\right]+o(1) \\
\geq \frac{1}{2}\left|\int_{Q} h\right||\ln s|+\frac{1}{2} \int_{Q}\left(\nabla \times A-h_{\mathrm{ex}}\right)^{2}+o\left(|\ln s|^{2}\right) .
\end{gathered}
$$

We may now resolve the minimization problem for parallel applied fields of magnitude $h_{\mathrm{ex}}=H_{\mathrm{ex}}|\ln s|$. After normalizing the magnetic fields $h_{s}=\nabla \times A$ by letting

$$
H_{s}=h_{s} /|\ln s|
$$

and applying the upper bound (4.3), we conclude that $H_{s}-H_{\mathrm{ex}}$ is uniformly bounded in $L^{2}$ and hence a subsequence $H_{s_{k}} \rightarrow H_{*}$ is weakly convergent. Passing to the limit using the upper bound (4.3) and (4.19) we have

$$
\begin{aligned}
\Phi(H):=\frac{1}{2}\left[|H|+\left(H-H_{\mathrm{ex}}\right)^{2}\right] & \geq \liminf _{s \rightarrow 0} \frac{\mathcal{L}_{\epsilon, s}\left(u_{n}, A\right)}{|Q||\ln s|^{2}} \\
& \geq \frac{1}{2} \frac{1}{|Q|}\left[\left|\int_{Q} H_{*}\right|+\int_{Q}\left(H_{*}-H_{\mathrm{ex}}\right)^{2}\right],
\end{aligned}
$$

which holds for every constant $H \in \mathbb{R}$. Note that the right-hand side is strictly convex, and thus it may be bounded below by replacing $H_{*}$ by its average value over the square $Q$, $\bar{H}_{*}=|Q|^{-1} \int_{Q} H_{*}$. Choosing the constant $H$ to minimize the left-hand expression, we have

$$
\min _{H \in \mathbb{R}} \Phi(H) \geq \frac{1}{2}\left[\left|\bar{H}_{*}\right|+\left(\bar{H}_{*}-H_{\mathrm{ex}}\right)^{2}\right]=\Phi\left(\bar{H}_{*}\right) .
$$

By the strict convexity of $\Phi: \mathbb{R} \rightarrow \mathbb{R}$ we conclude that $\bar{H}_{*}$ minimizes $\Phi$, and since there is equality in (4.20) we further conclude that $H_{*}=\bar{H}_{*}$ is constant and minimizes $\Phi$. A simple calculation shows that this minimum is given by $H_{*}=0$ if $0 \leq H_{\mathrm{ex}} \leq 1 / 2$ and $H_{*}=H_{\mathrm{ex}}-1 / 2$ if $H_{\mathrm{ex}} \geq 1 / 2$. Thus, in physicist's terms, to highest order in $s$ the lower critical field in the parallel case is $H_{C 1}=\frac{1}{2}|\ln s|$. This concludes the proof of Theorem 1.3 in the case $h_{\mathrm{ex}}=O(|\ln s|)$.

We now consider the case $|\ln s| \ll h_{\mathrm{ex}} \ll s^{-2}$. For strong applied fields the vortex ball construction may not be applied directly, and we require a different approach, as in Chapter 8 of [SS].

Proposition 4.8. Assume $|\ln s| \ll h_{\mathrm{ex}} \ll s^{-2}$, $\left(u_{n}, A\right)$ minimizes $\mathcal{L}_{\epsilon, s}$, and $\Psi$ is given as in (4.11). Then, for every constant $K>0$, there exists $\lambda$ with $1 \ll \lambda \ll 1 / s$ and $\beta_{K}>0$ such that for every ball $B_{y}^{\lambda}:=B\left(y, \lambda^{-1}\right)$ we have, as $s \rightarrow 0$,

$$
\mathcal{M}_{s}\left(\Psi, A ; B_{y}^{\lambda}\right) \geq \frac{\beta_{K}\left|B_{y}^{\lambda}\right|}{2} h_{\mathrm{ex}} \ln \left(\frac{1}{s \sqrt{h_{\mathrm{ex}}}}\right)(1-o(1)),
$$

and $\beta_{K} \rightarrow 1$ as $K \rightarrow \infty$ 
Proof. We provide a sketch, as the steps follow those in Proposition 8.2 of [SS]. Without loss, we take the center of the ball to be the origin, $y=0$. First, for any fixed positive $\lambda$ we rescale, $\Psi_{\lambda}(\lambda x)=\Psi(x), \lambda A_{\lambda}(\lambda x)=A(x), s^{\prime}=\lambda s$, and $h_{\mathrm{ex}}^{\prime}=h_{\mathrm{ex}} \lambda^{-2}$. Introducing a rescaled energy, we have

$$
\begin{aligned}
\mathcal{M}_{s}\left(\Psi, A ; B_{0}^{\lambda}\right)= & \mathcal{M}_{s^{\prime}}^{\lambda}\left(\Psi_{\lambda}, A_{\lambda} ; B_{0}^{1}\right) \\
= & \frac{1}{2} \int_{B_{0}^{1}}\left\{\left|\Psi_{\lambda}\right|^{2}\left|\nabla_{A_{\lambda}} \Psi_{\lambda}\right|^{2}+\lambda^{2}\left(\operatorname{curl} A_{\lambda}-h_{\mathrm{ex}}^{\prime}\right)^{2}\right. \\
& \left.+\frac{1}{2}\left(1-\left|\Psi_{\lambda}\right|^{2}\right)\left[|\nabla| \Psi_{\lambda}||^{2}+\frac{\alpha}{\left(s^{\prime}\right)^{2}}\left(1-\left|\Psi_{\lambda}\right|^{2}\right)^{2}\right]\right\} .
\end{aligned}
$$

As in (8.16) of [SS], it is possible to choose $\lambda$ such that

$$
h_{\mathrm{ex}}^{\prime}=K\left|\ln s^{\prime}\right|, \quad 1 \ll \lambda \ll s^{-1}, \quad s^{\prime} \ll 1, \quad \ln \frac{1}{s \sqrt{h_{\mathrm{ex}}}} \sim\left|\ln s^{\prime}\right| .
$$

Thus, in the new coordinates we return to the small-field case above, in which the vortex ball construction is valid, and we seek to show that, as $s^{\prime} \rightarrow 0$,

$$
\mathcal{M}_{s^{\prime}}^{\lambda}\left(\Psi_{\lambda}, A_{\lambda} ; B_{0}^{1}\right) \geq \frac{\beta_{K}\left|B_{0}^{1}\right|}{2} h_{\mathrm{ex}}^{\prime}\left|\ln s^{\prime}\right|(1-o(1))
$$

for some $\beta_{K}>0$. We may assume that, for some constant $C>0$,

$$
\mathcal{M}_{s^{\prime}}^{\lambda}\left(\Psi_{\lambda}, A_{\lambda} ; B_{0}^{1}\right) \leq C\left(h_{\mathrm{ex}}^{\prime}\right)^{2}
$$

for otherwise (4.22) certainly holds for any constant $\beta_{K}$.

The next step is to bound $\mathcal{M}_{s^{\prime}}^{\lambda}\left(\Psi_{\lambda}, A_{\lambda} ; B_{0}^{1}\right)$ from below by the minimum of $\mathcal{M}_{s}$ in $H^{1}\left(B_{0}^{1}\right)$ (that is, with natural boundary conditions on $\partial B_{0}^{1}$, rather than periodic). This follows the analogous argument for the Ginzburg-Landau functional, using the ball construction (Theorem 4.6) and weak convergence of the rescaled fields and currents outside of the vortex balls, as in the proof of item 1 of Theorem 7.1 in [SS]. The crucial observation is that outside of the vortex balls, $\mathcal{M}_{s}$ agrees with Ginzburg-Landau (with $s$ replacing $\epsilon$ ) to highest order, and so the same lower bound may be obtained:

$$
\liminf _{s^{\prime} \rightarrow 0} \frac{\mathcal{M}_{s^{\prime}}\left(\Psi_{\lambda}, A_{\lambda} ; B_{0}^{1}\right)}{\left(h_{\mathrm{ex}}^{\prime}\right)^{2}} \geq \min _{\mu} E_{K}(\mu),
$$

with

$$
E_{K}(\mu)=\frac{\|\mu\|}{2 K}+\frac{1}{2} \int_{B_{0}^{1}}\left[\left|\nabla h_{\mu}\right|^{2}+\left|h_{\mu}-1\right|^{2}\right]
$$

defined for measures $\mu \in H^{-1}$, where $h_{\mu}$ solves

$$
-\Delta h_{\mu}+h_{\mu}=\mu,\left.\quad h_{\mu}\right|_{\partial B_{0}^{1}}=1
$$


The minimizers correspond to the solutions of an obstacle problem, and for the ball an explicit solution is given in (7.15) of [SS]. In particular,

$$
\min _{\mu} E_{K} \geq \frac{1}{2 K}\left(1-\frac{1}{2 K}\right)\left|\omega_{K}\right|
$$

with coincidence set $\omega_{K} \rightarrow B_{0}^{1}$ as $K \rightarrow \infty$. Using the relations (4.21), we obtain (4.22) with $\beta_{K}=\left(1-\frac{1}{2 K}\right)\left|\omega_{K}\right| /\left|B_{0}^{1}\right| \rightarrow 1$ as $K \rightarrow \infty$.

We may now complete the proof of the second part of Theorem 1.3. Consider the energy density associated to $\mathcal{M}_{S}(\Psi, A)$ as a measure,

$d v_{s}=\frac{d x}{h_{\mathrm{ex}} \ln \frac{1}{s \sqrt{h_{\mathrm{ex}}}}}\left\{|\Psi|^{2}\left|\left(\nabla_{A}\right) \Psi\right|^{2}+\frac{1}{2}\left(1-|\Psi|^{2}\right)\left(|\nabla| \Psi||^{2}+\frac{\alpha}{s^{2}}\left(1-|\Psi|^{2}\right)^{2}\right)+\left(h-h_{\mathrm{ex}}\right)^{2}\right\}$.

By the upper bound (Lemma 4.4), $v_{s}$ is a bounded family and along a subsequence converges weakly in the sense of measures, $v_{s} \rightarrow v$. Proceeding as in [SS], given any open set $U$, integrating the lower bound (from Proposition 4.8) over the centers $y \in U$ we obtain

$$
\liminf _{s \rightarrow 0} v_{s}(U)=\liminf _{s \rightarrow 0} \frac{\mathcal{M}_{s}(\Psi, A ; U)}{h_{\mathrm{ex}} \ln \frac{1}{s \sqrt{h_{\mathrm{ex}}}}} \geq \frac{|U|}{2} .
$$

Consequently, $v \geq \frac{1}{2} d x$, and from the upper bound we must have equality. This proves the first assertion of item 2 . To conclude, note that

$$
\left\|h_{s} / h_{\mathrm{ex}}-1\right\|_{L^{2}}^{2} \leq \mathcal{M}_{s}(\Psi, A) / h_{\mathrm{ex}}^{2} \rightarrow 0
$$

since $\ln \frac{1}{s \sqrt{h_{\mathrm{ex}}}} \ll h_{\mathrm{ex}}$.

Case $s^{2} h_{\mathrm{ex}} \gg 1$

In case $s^{2} h_{\mathrm{ex}} \gg 1$ the minimizers of the Lawrence-Doniach functional in a parallel applied field behave quite differently from those of the Ginzburg-Landau model. As we will see, there is no "upper critical field" at which superconductivity is diminished and eventually extinguished, but instead a "transparent phase" in which the applied field penetrates the sample completely with no apparent loss of superconductivity in the planes.

It will be convenient to permit some variation in the period domain, $Q=\left[0, L_{1}\right] \times$ $\left[0, L_{3}\right]$, and in fact we will see that energy minimization reveals a subtle relationship between the period and the applied field $h_{\mathrm{ex}}$.

We assume throughout that $\left(u_{n}, A\right) \in \mathcal{H}$ are minimizers of $\mathcal{L}_{\epsilon, s}$. Recall the definitions of $\rho, J_{x}$ from (1.4) in the introduction,

$$
\begin{aligned}
\rho(x, z) & =\sum_{n=1}^{N}\left|u_{n}(x)\right| \chi_{\left(z_{n-1}, z_{n}\right]}(z) \\
J_{x}(x, z) & =\sum_{n=1}^{N}\left(i u_{n}(x), u_{n}^{\prime}-i A_{x}\left(x, z_{n}\right)\right) \chi_{\left(z_{n-1}, z_{n}\right]}(z) .
\end{aligned}
$$


In some sense, $\rho$ measures the superconducting density, and $J_{x}$ the horizontal currents, extended to the entire bulk. The interpolating function $\Psi$ used in the previous regime cannot be easily related to the energy density, as the control on the error terms in the finite differences in $x$ is lost in this highly oscillatory limit.

Proof of Theorem 1.4. We first recall some facts about fixing a gauge in $\mathcal{H}$ in the parallel field setting. For a constant field $\bar{h} \in \mathbb{R}$, we fix the representative vector potential,

$$
\bar{A}=\left(\bar{A}_{x}, \bar{A}_{z}\right)=\frac{\bar{h}}{2}(z,-x) .
$$

As in (2.1), (2.2) we say $\left(u_{n}, A\right) \in \mathcal{H}_{*}$ if $u_{n} \in H_{\text {loc }}^{1}(\mathbb{R} ; \mathbb{C})$ for all $n \in \mathbb{Z}, A \in$ $H_{\text {loc }}^{1}\left(\mathbb{R}^{2} ; \mathbb{R}^{2}\right)$, and there exists a constant number $\bar{h} \in \mathbb{R}$ so that

$$
\left\{\begin{array}{l}
A=\bar{A}+\hat{A}, \quad \operatorname{div} \hat{A}=0, \quad \hat{A}\left(x+L_{1}, z\right)=\hat{A}(x, z)=\hat{A}(x, z+1), \\
u_{n}\left(x+L_{1}\right)=u_{n}(x) e^{-i \bar{h} z_{n} L_{1} / 2}, \quad u_{n+N}(x)=u_{n}(x) e^{i \bar{h} x L_{3} / 2}, \quad \forall n \in \mathbb{Z} .
\end{array}\right.
$$

Since by Green's theorem we must have $\int_{Q} \operatorname{curl} \hat{A}=0$, it follows that the constant $\bar{h}$ is the mean value of $h=\operatorname{curl} A$ over the period domain $Q$. By Lemma 2.1, for any configuration $\left(\tilde{u}_{n}, \tilde{A}\right) \in \mathcal{H}$ there exists $\lambda \in H_{\text {loc }}^{2}$ so that $\left(u_{n}, A\right)=\left(\tilde{u}_{n} e^{i \lambda\left(\cdot, z_{n}\right)}, \tilde{A}+\nabla \lambda\right) \in \mathcal{H}_{*}$. Furthermore, from (2.3) we conclude that there exists a constant $C_{0}$ with

$$
\|\hat{A}\|_{H^{1}(Q)} \leq C_{0}\|\operatorname{curl} \hat{A}\|_{L^{2}(Q)}
$$

for any $\left(u_{n}, A\right) \in \mathcal{H}_{*}$.

Upper bound. We obtain an upper bound on the energy by choosing a configuration $\left(v_{n}, \bar{A}\right)$ with $\bar{A}=\frac{1}{2} \bar{h}(z,-x), \bar{h}$ constant, and $v_{n}=e^{i \phi_{n}}$ with $\phi_{n}(x)=\frac{1}{2} \bar{h} z_{n} x, n \in \mathbb{Z}$. Note that with this choice, $v_{n}^{\prime}-i \bar{A}_{x}\left(x, z_{n}\right) v_{n}=0$. A simple calculation shows that $\left(v_{n}, \bar{h}\right) \in \mathcal{H}_{*}$ provided $\bar{h} s L_{1} \in 2 \pi \mathbb{Z}$. Hence, recalling that $s N=L_{3}$,

$$
\min _{\mathcal{H}_{*}} \mathcal{L}_{\epsilon, s} \leq \frac{1}{2 s} \sum_{n=1}^{N} \int_{0}^{L_{1}} 2[1-\cos (\bar{h} s x)] d x+\frac{1}{2}|Q|\left(\bar{h}-h_{\mathrm{ex}}\right)^{2}=\frac{|Q|}{s^{2}}+\frac{1}{2}|Q|\left(\bar{h}-h_{\mathrm{ex}}\right)^{2} .
$$

By minimizing the right-hand side over $\bar{h} \in \frac{2 \pi}{s L_{1}} \mathbb{Z}$ we obtain the upper bound in the generic case,

$$
\min _{\mathcal{H}_{*}} \mathcal{L}_{\epsilon, s} \leq \frac{|Q|}{s^{2}}+\frac{1}{2} \min _{\bar{h} \in \frac{2 \pi}{s L_{1}} \mathbb{Z}}|Q|\left(\tilde{h}-h_{\mathrm{ex}}\right)^{2} \leq \frac{|Q|}{s^{2}}\left(1+\frac{\pi^{2}}{2 L_{1}^{2}}\right)+o\left(s^{-2}\right) .
$$

Of course, in the special case where $h_{\mathrm{ex}} \in \frac{2 \pi}{s L_{1}} \mathbb{Z}$, the sharper bound prevails,

$$
\min _{\mathcal{H}_{*}} \mathcal{L}_{\epsilon, s} \leq \frac{|Q|}{s^{2}}+o\left(s^{-2}\right) .
$$

Lower bound. We next derive a lower bound on minimizers $\left(u_{n}, A\right) \in \mathcal{H}_{*}$. The idea is to try to mimic the test function in the upper bound as closely as possible. Decompose $A=$ 
$\bar{A}+\hat{A}$ as above with $\bar{A}=\frac{1}{2} h_{\mathrm{ex}}(-z, x)$ and define a family of phases $\phi_{n}(x)=\frac{1}{2} h_{\mathrm{ex}} z_{n} x$, $n \in \mathbb{Z}$. Now define

We observe that

$$
v_{n}=u_{n} e^{-i \phi_{n}} .
$$

$$
\begin{gathered}
\left|v_{n}\right|=\left|u_{n}\right|, \quad\left|v_{n}^{\prime}-i \hat{A}_{x}\left(x, z_{n}\right) v_{n}\right|=\left|u_{n}^{\prime}-i A_{x}\left(x, z_{n}\right) u_{n}\right|, \\
\left(i u_{n}, u_{n}^{\prime}-i A_{x}\left(x, z_{n}\right) u_{n}\right)=\left(i v_{n}, v_{n}^{\prime}-i \hat{A}_{x}\left(x, z_{n}\right) v_{n}\right) .
\end{gathered}
$$

In terms of $v_{n}$ and $\hat{A}$, we have

$$
\begin{aligned}
\mathcal{L}_{\epsilon, S}\left(u_{n}, A\right)= & s \sum_{n=1}^{N} \int_{0}^{L_{1}}\left(\frac{1}{2}\left|v_{n}^{\prime}-i \hat{A}_{x}\left(x, z_{n}\right) v_{n}\right|^{2}+\frac{1}{4 \epsilon^{2}}\left(\left|v_{n}\right|^{2}-1\right)^{2}\right) \\
& +\frac{1}{2 s} \sum_{n=1}^{N} \int_{0}^{L_{1}}\left|v_{n}-v_{n-1} \exp \left\{i \Phi_{n}(x)\right\}\right|^{2}+\frac{1}{2} \int_{Q} \hat{h}^{2},
\end{aligned}
$$

where $\hat{h}:=\operatorname{curl} \hat{A}$ and

$$
\Phi_{n}(x):=-h_{\mathrm{ex}} s x+\int_{z_{n-1}}^{z_{n}} \hat{A}_{z}(x, z) d z .
$$

The Josephson coupling term will provide the desired lower bound. We expand it:

$$
\begin{aligned}
\left|v_{n}-v_{n-1} e^{i \Phi_{n}}\right|^{2} & =\left|v_{n}\right|^{2}+\left|v_{n-1}\right|^{2}-2 \operatorname{Re}\left\{v_{n} \overline{v_{n-1}} e^{-i \Phi_{n}}\right\} \\
& =2-\left(1-\left|v_{n}\right|^{2}\right)-\left(1-\left|v_{n-1}\right|^{2}\right)-2 \operatorname{Re}\left\{v_{n} \overline{v_{n-1}} e^{-i \Phi_{n}}\right\} \\
& \geq 2-\frac{s^{2}}{8 \epsilon^{2}}\left[\left(1-\left|v_{n}\right|^{2}\right)+\left(1-\left|v_{n-1}\right|^{2}\right)\right]-\frac{4 \epsilon^{2}}{s^{2}}-2 \operatorname{Re}\left\{v_{n} \overline{v_{n-1}} e^{-i \Phi_{n}}\right\},
\end{aligned}
$$

applying the inequality $x \leq \delta x^{2}+1 / 4 \delta$ with $\delta=s^{2} / 8 \epsilon^{2}$ to bound $x=1-\left|v_{n}\right|^{2}$. It follows that

$$
\begin{aligned}
\mathcal{L}_{\epsilon, s}\left(u_{n}, A\right) \geq & s \sum_{n=1}^{N} \int_{0}^{L_{1}}\left(\frac{1}{2}\left|v_{n}^{\prime}-i \hat{A}_{x}\left(x, z_{n}\right) v_{n}\right|^{2}+\frac{1}{8 \epsilon^{2}}\left(\left|v_{n}\right|^{2}-1\right)^{2}\right) \\
& +s \sum_{n=1}^{N} \int_{0}^{L_{1}} \frac{1}{s^{2}} d x+\frac{1}{2} \int_{Q} \hat{h}^{2}-s \sum_{n=1}^{N} \int_{0}^{L_{1}} \frac{\operatorname{Re}\left\{v_{n} \overline{v_{n-1}} e^{-i \Phi_{n}}\right\}}{s^{2}} .
\end{aligned}
$$

We use integration by parts to estimate the last term, which is the only potentially negative one:

$$
\begin{aligned}
\int_{0}^{L_{1}} v_{n} \overline{v_{n-1}} e^{-i \Phi_{n}} & =\int_{0}^{L_{1}}\left[v_{n} \overline{v_{n-1}} e^{-i \int_{z_{n-1}}^{z_{n}} \hat{A}_{z} d z}\right] e^{i h_{\mathrm{ex}} s x} d x \\
= & \frac{1}{h_{\mathrm{ex}} s}\left[v_{n} \overline{v_{n-1}} e^{-i \Phi_{n}}\right]_{0}^{L_{1}} \\
& -\frac{1}{h_{\mathrm{ex}} s} \int_{0}^{L_{1}}\left(v_{n}^{\prime} \overline{v_{n-1}}+v_{n} \overline{v_{n-1}^{\prime}}-i v_{n} \overline{v_{n-1}} \int_{z_{n-1}}^{z_{n}} \partial_{x} \hat{A}_{z} d z\right) e^{-i \Phi_{n}} d x .
\end{aligned}
$$


It follows, using $\left|v_{n}\right| \leq 1$ from Proposition 2.2 and the equality

$$
\int_{z_{n-1}}^{z_{n}} \partial_{x} \hat{A}_{z} d z=\int_{z_{n-1}}^{z_{n}}\left(\hat{h}+\partial_{z} \hat{A}_{x}\right)=\hat{A}_{x}\left(z_{n}\right)-\hat{A}_{z}\left(z_{n-1}\right)+\int_{z_{n-1}}^{z_{n}} \hat{h}
$$

that

$$
\begin{aligned}
& \left|\int_{0}^{L_{1}} v_{n} \overline{v_{n-1}} e^{-i \Phi_{n}}\right| \leq \frac{2}{h_{\mathrm{ex}} s} \\
& \quad+\frac{1}{h_{\mathrm{ex}} S} \int_{0}^{L_{1}}\left(\left|v_{n}^{\prime}-i \hat{A}_{x}\left(x, z_{n}\right) v_{n}\right|+\left|v_{n-1}^{\prime}-i \hat{A}_{x}\left(x, z_{n-1}\right) v_{n-1}\right|+\int_{z_{n-1}}^{z_{n}}|\hat{h}| d z\right) d x .
\end{aligned}
$$

In particular,

$$
\begin{aligned}
\frac{1}{s^{2}}\left|\int_{0}^{L_{1}} v_{n} \overline{v_{n-1}} e^{-i \Phi_{n}}\right| \leq \frac{2}{h_{\mathrm{ex}} s^{3}}+\int_{0}^{L_{1}}\left[\left(\frac{\left|v_{n}^{\prime}-i \hat{A}_{x}\left(x, z_{n}\right) v_{n}\right|^{2}}{8}+\frac{8}{h_{\mathrm{ex}}^{2} s^{6}}\right)\right. \\
\left.+\left(\frac{\left|v_{n-1}^{\prime}-i \hat{A}_{x}\left(x, z_{n-1}\right) v_{n-1}\right|^{2}}{8}+\frac{8}{h_{\mathrm{ex}}^{2} s^{6}}\right)+\left(\frac{1}{4} \int_{z_{n-1}}^{z_{n}} \hat{h}^{2} d z+\frac{s}{h_{\mathrm{ex}}^{2} s^{6}}\right)\right] d x .
\end{aligned}
$$

Together with (4.30) we find

$$
\begin{aligned}
\mathcal{L}_{\epsilon, s}\left(u_{n}, A\right) \geq & s \sum_{n=1}^{N} \int_{0}^{L_{1}} \frac{1}{s^{2}} d x \\
& +s \sum_{n=1}^{N} \int_{0}^{L_{1}}\left[\frac{\left|v_{n}^{\prime}-i \hat{A}_{x}\left(x, z_{n}\right) v_{n}\right|^{2}}{4}+\frac{1}{8 \epsilon^{2}}\left(1-\left|v_{n}\right|^{2}\right)^{2}\right] d x \\
& +\frac{1}{4} \int_{Q} \hat{h}^{2}-\left\{\frac{2 L_{3}}{h_{\mathrm{ex}} s^{3}}+\frac{16 L_{1} L_{3}}{h_{\mathrm{ex}}^{2} s^{6}}+\frac{s L_{3}}{h_{\mathrm{ex}}^{2} s^{6}}\right\} .
\end{aligned}
$$

The expression in braces is $o\left(1 / s^{2}\right)$, using the fact that $h_{\mathrm{ex}} s^{2}=o(1)$ and $s=o(1)$.

Then, with no assumption on $h_{\mathrm{ex}}, L_{1}$ we obtain (1.5), while if $h_{\mathrm{ex}} \in \frac{2 \pi N}{|Q|} \mathbb{Z}$, then the matching upper bound $\mathcal{L}_{\epsilon, s}\left(u_{n}, A\right) \leq|Q| / s^{2}+o\left(1 / s^{2}\right)$ holds and therefore, using (4.27) and the notation (1.4),

$$
\int_{Q}\left(J_{x}^{2}+\frac{1}{4 \epsilon^{2}}\left(\rho^{2}-1\right)^{2}+\frac{1}{2}\left(h-h_{\mathrm{ex}}\right)^{2}\right)=o\left(s^{-2}\right) .
$$

This concludes the proof of Theorem 1.4.

Acknowledgments. The research of S. Alama was supported by an NSERC Research Grant. 


\section{References}

[ABB1] Alama, S., Berlinsky, A. J., Bronsard, L.: Minimizers of the Lawrence-Doniach energy in the small-coupling limit: finite width samples in a parallel field. Ann. Inst. H. Poincaré Anal. Non Linéaire 19, 281-312 (2002) Zbl 1011.82032 MR 1956952

[ABB2] Alama, S., Bronsard, L., Berlinsky, A. J.: Periodic vortex lattices for the LawrenceDoniach model of layered superconductors in a parallel field, Comm. Contemp. Math. 3, 457-494 (2001) Zbl 1006.82040 MR 1849651

[ABS1] Alama, S., Bronsard, L., Sandier, E.: On the shape of interlayer vortices in the Lawrence-Doniach model. Trans. Amer. Math. Soc. 360, 1-34 (2008) Zbl 1132.35081 MR 2341992

[ABS2] Alama, S., Bronsard, L., Sandier, E.: Periodic minimizers of the anisotropic Ginzburg-Landau model. Calc. Var. Partial Differential Equations 36, 399-417 (2009) Zbl 1180.35494 MR 2551137

[AyS] Aydi, H., Sandier, E.: Vortex analysis of the periodic Ginzburg-Landau model. Ann. Inst. H. Poincaré Anal. Non Linéaire 26, 1223-1236 (2009) Zbl 1171.35480 MR 2542722

[BaK] Bauman, P., Ko, Y.: Analysis of solutions to the Lawrence-Doniach system for layered superconductors. SIAM J. Math. Anal. 37, 914-940 (2005) Zbl 1141.82019 MR 2191782

[BBO] Bethuel, F., Brezis, H., Orlandi, G.: Asymptotics for the Ginzburg-Landau equation in arbitrary dimensions. J. Funct. Anal. 186, 432-520 (2001) Zbl 1077.35047 MR 1864830

[BuCm] Bulaevskiǔ, L., Clem, J.: Vortex lattice of highly anisotropic layered superconductors in strong, parallel magnetic fields. Phys. Rev. B 44, 10234-10238 (1991)

[Cl] Clem, J.: Two-dimensional vortices in a stack of thin superconducting films: A model for high-temperature superconducting multilayers. Phys. Rev. B 43, 7837-7846 (1991)

[DGP] Du, Q., Gunzburger, M. D., Peterson, J. S.: Analysis and approximation of the GinzburgLandau model of superconductivity. SIAM Rev. 34, 54-81 (1992) Zbl 0787.65091 MR 1156289

[Je] Jerrard, R.: Lower bounds for generalized Ginzburg-Landau functionals. SIAM J. Math. Anal. 30, 721-746 (1999) Zbl 0928.35045 MR 1684723

[LaDo] Lawrence, W., Doniach, S.: Theory of layer structure superconductors. In: Proc. 12th Int. Conf. on Low Temperature Physics, E. Kanda (ed.), Academic Press of Japan, Kyoto, 361-362 (1971)

[Sa] Sandier, E.: Lower bounds for the energy of unit vector fields and applications. J. Funct. Anal. 152, 379-403 (1998) Zbl 0908.58004 MR 1742866

[SS] Sandier, E., Serfaty, S.: Vortices in the Magnetic Ginzburg-Landau Model. Progr. Nonlinear Differential Equations Appl. 70, Birkhäuser Boston (2007) Zbl 1112.35002 MR 2279839 